\title{
Updated report on tools to measure outcomes of clinical trials in fragile $X$ syndrome
}

Dejan B. Budimirovic ${ }^{1 *}$, Elizabeth Berry-Kravis ${ }^{2}$, Craig A. Erickson ${ }^{3}$, Scott S. Hall ${ }^{4}$, David Hess ${ }^{5}$, Allan L. Reiss ${ }^{6}$, Margaret K. King ${ }^{7}$, Leonard Abbeduto ${ }^{8}$ and Walter E. Kaufmann ${ }^{9,10^{*}}$

\begin{abstract}
Objective: Fragile X syndrome (FXS) has been the neurodevelopmental disorder with the most active translation of preclinical breakthroughs into clinical trials. This process has led to a critical assessment of outcome measures, which resulted in a comprehensive review published in 2013. Nevertheless, the disappointing outcome of several recent phase III drug trials in FXS, and parallel efforts at evaluating behavioral endpoints for trials in autism spectrum disorder (ASD), has emphasized the need for re-assessing outcome measures and revising recommendations for FXS.
\end{abstract}

Methods: After performing an extensive database search (PubMed, Food and Drug Administration (FDA)/National Institutes of Health (NIH)'s www.ClinicalTrials.gov, etc.) to determine progress since 2013, members of the Working Groups who published the 2013 Report evaluated the available outcome measures for FXS and related neurodevelopmental disorders using the COSMIN grading system of levels of evidence. The latter has also been applied to a British survey of endpoints for ASD. In addition, we also generated an informal classification of outcome measures for use in FXS intervention studies as instruments appropriate to detect shorter- or longer-term changes.

Results: To date, a total of 22 double-blind controlled clinical trials in FXS have been identified through www. ClinicalTrials.gov and an extensive literature search. The vast majority of these FDA/NIH-registered clinical trials has been completed between 2008 and 2015 and has targeted the core excitatory/inhibitory imbalance present in FXS and other neurodevelopmental disorders. Limited data exist on reliability and validity for most tools used to measure cognitive, behavioral, and other problems in FXS in these trials and other studies. Overall, evidence for most tools supports a moderate tool quality grading. Data on sensitivity to treatment, currently under evaluation, could improve ratings for some cognitive and behavioral tools. Some progress has also been made at identifying promising biomarkers, mainly on blood-based and neurophysiological measures.

\footnotetext{
* Correspondence: dbudimi1@jhu.edu; wkaufmann@ggc.org

${ }^{1}$ Departments of Psychiatry and Behavioral Sciences, Kennedy Krieger

Institute and Child Psychiatry, Johns Hopkins University School of Medicine,

716 N. Broadway, Baltimore, MD 21205, USA

${ }^{9}$ Center for Translational Research, Greenwood Genetic Center, 113 Gregor

Mendel Circle, Greenwood, SC 29646, USA

Full list of author information is available at the end of the article
} 


\begin{abstract}
(Continued from previous page)
Conclusion: Despite the tangible progress in implementing clinical trials in FXS, the increasing data on measurement properties of endpoints, and the ongoing process of new tool development, the vast majority of outcome measures are at the moderate quality level with limited information on reliability, validity, and sensitivity to treatment. This situation is not unique to FXS, since reviews of endpoints for ASD have arrived at similar conclusions. These findings, in conjunction with the predominance of parent-based measures particularly in the behavioral domain, indicate that endpoint development in FXS needs to continue with an emphasis on more objective measures (observational, direct testing, biomarkers) that reflect meaningful improvements in quality of life. A major continuous challenge is the development of measurement tools concurrently with testing drug safety and efficacy in clinical trials.
\end{abstract}

Keywords: Fragile X syndrome, Clinical trials, Outcome measures, Intellectual disability, Autism spectrum disorder

\section{Background}

Fragile X syndrome (FXS) is caused by an unstable expansion of a polymorphic trinucleotide (CGG) repeat sequence in the regulatory region of the fragile $\mathrm{X}$ mental retardation 1 gene (FMR1), which leads to its epigenetic silencing via atypical methylation [1]. This results in a deficit of the FMR1-encoded protein fragile $\mathrm{X}$ mental retardation protein (FMRP), an RNA-binding protein that regulates dendritic translation and plays a critical role in synaptic development and function $[1,2]$. With a prevalence of about $1 / 4000$, FXS is a relatively common single monogenic cause of intellectual disability (ID) and autism spectrum disorder (ASD). Patients with FXS experience a wide range of cognitive, behavioral, and systemic manifestations that often require a complex array of behavioral and pharmacological interventions [2, 3]. A number of studies of symptom-based pharmacological treatments in individuals with FXS have been performed in small cohorts or without randomization or blinding (reviewed in [4]). Thus, as for other neurodevelopmental disorders with behavioral symptoms, guidance is somewhat lacking regarding the effectiveness of targeting clinical manifestations of FXS [e.g., attention-deficit hyperactivity disorder (ADHD)]. Nonetheless, advances in experimental models of FXS and other neurodevelopmental disorders with known genetic bases have opened the door for the development of disease- and neurobiological mechanism-specific pharmacological treatments [5].

From the beginning of the era of neurobiological "targeted" trials in FXS, there have been concerns about endpoints. Thus, the National Institutes of Health (NIH) convened two meetings of leading scientists and clinicians, which included other key stakeholders (e.g., Food and Drug Administration (FDA), advocacy groups, pharmaceutical industry) to discuss the subject of outcome measures. The second and key meeting, held in 2009, led to the creation of Outcome Measures Working Groups (here termed Working Groups) and a publication summarizing the findings of these Groups [6]. Problems identified during these meetings included the following: (1) few measures were validated for FXS; (2) investigators used different measures due to lack of consensus; (3) measures were unable to cover multiple levels of function; (4) limited number of measures were validated or standardized for ID populations, with many having substantial floor effects; (5) measures involving direct observation were lacking (i.e., most instruments were rating scales); and (6) biomarkers that quantify brain function, viewed as the most useful biomarkers, had demonstrated clear differences between FXS and control individuals but none had well-established clinical correlations or validity [6]. To address these shortcomings, at the 2009 meeting, the Working Groups were tasked with identifying outcome measures in three areas relevant to the broad phenotype of FXS: (1) Cognition, (2) Behavior/Emotion, and (3) Medical/Physical including biomarkers. Their work was guided by three principles: (1) identification of a core set of widely applicable measures would facilitate comparability across different agents, research centers, and methodological approaches; (2) outcome measures should be validated specifically for FXS since those that have been developed for symptom-based clinical trials in behaviorally defined disorders (e.g., ADHD) might not be sufficiently sensitive or specific; and (3) the Working Groups should generate a set of criteria for identifying appropriate outcome measures for design, interpretation, and guiding funding of clinical trials in FXS [6]. In addition to specific recommendations for each of the three areas, the Working Groups concluded that (1) research on biomarkers for detecting treatment response in FXS was in its infancy, but this was an area of utmost importance; (2) measures under development have to be linked to the neurobiology of FXS; and (3) continued research is greatly needed in multiple areas, including the core behavioral field.

Since 2009, there has been an explosion of clinical trials in FXS, especially targeted drug interventions (reviewed in [7]). Despite adequate power and other study design strengths, successful phase II trials that advanced to phase III failed to demonstrate therapeutic efficacy based on their primary endpoints $[8,9]$. This has raised questions about the promise of translating breakthrough drug studies in mouse and other experimental models to humans with FXS. Although the ongoing analysis of methodological 
issues underlying these failures has identified multiple possible explanations, inadequacy of available biomarkers and outcome measures is unquestionably a factor [8]. This situation has led to a re-assessment of the quality of existing outcome measures in FXS and the need to update the recommendations from the Working Groups in the 2013 Report [6]. The perceived "crisis" in the FXS treatment field also represents an opportunity to implement novel study designs and methodologies. An example of this is an upcoming trial re-examining the efficacy of the metabotropic glutamate receptor 5 (mGluR5) antagonist mavoglurant in FXS, which will introduce modifications such as the study of younger individuals, presumably more receptive to interventions targeting synaptic development, and the use of a cognitive intervention during the drug trial, aimed at enhancing drug effect on learning and consequently on synaptic plasticity. Introduction of novel outcome measures and examination of potential biomarkers already studied in mouse models (e.g., auditory evoked potentials) are also components of this innovative trial (U01 NS096767, Effects of AFQ056 on Language Learning in Young Children with Fragile X Syndrome, P.I. Berry-Kravis).

This report represents a re-assessment of outcome measures in FXS taking into account progress in the field since the 2013 publication. It preserves the principles (i.e., critical review and recommendations for measures currently available or under development) and structure (i.e., Cognition, Behavior/Emotion, Biomarkers/Medical types of outcome measures) of the 2013 Report. Nevertheless, it does also introduce new elements. These include the classification of measures in terms of their projected ability to detect shorter-term and/or longer-term changes and a grading of endpoints based on their measurement/psychometric properties using the COnsensus-based Standards for the selection of health Measurement INstruments (COSMIN) system (www.cosmin.nl). The latter was recently applied by the UK NHS' National Institute for Health Research to the evaluation of tools measuring outcomes in young children with ASD [10-12]. We hope this re-evaluation of outcome measures in FXS will provide guidance for the design and implementation of clinical trials (pharmacologic or other interventions) in this and other neurodevelopmental disorders.

\section{Material and methods}

The basis for our evaluation of available outcome measures was an extensive database search that included MEDLINE/PubMed, Embase, PsycINFO, and Web of Science, originally performed in December 2015 and rerun in January 2016. The search used keywords for individual outcome measures, including their reliability or validity in FXS, in general, and in clinical trials, in particular. For example, "ADI-R" AND "fragile X" OR
"ADI-R" AND "fragile X" AND "reliability," OR "ADI-R" AND "fragile X" AND "validity". If the data were limited or not available for FXS, an additional search included other fields of relevance (e.g., ASD, Rett syndrome). For instance, "ADI-R" AND "autism spectrum disorder" OR "ADI-R"“AND "autism spectrum disorder" AND "reliability," OR "ADI-R” AND "autism spectrum disorder" AND "validity". In addition to these systematic searches, several authors applied their experience with the use or development of certain measures for trials involving FXS or other neurodevelopmental disorders.

After dividing Cognition, Behavior/Emotion, and Biomarker/Medical (Medical/Physical in the 2013 Report) outcome measures into distinct subdomains, we generated an expert consensus on their classification as shorter- and/ or longer-term outcome/effect endpoints. An initial criterion for the shorter-term grouping was experience with the measures in FXS placebo-controlled trials lasting less than 12 months (see Table 1). On the other hand, a measure was considered as longer-term outcome on the basis of its use in open-label extensions of controlled clinical trials, the majority of them lasting 12 months or longer [7]. Due to limited empirical evidence, these criteria were complemented by expert agreement on particular types of function and the time necessary for detecting change. Because of their temporal dimension, typically at least 1 year, longer-term outcomes tend to reflect more global levels of function in particular in the cognitive domain (e.g., crystallized knowledge evaluated by intelligence and academic skills tests, adaptive behavior skills). Using the same logic, some types of manifestations, particularly problem behaviors, could change over short or long time periods. Thus, most behavioral measures could be either shorter- or longer-term outcomes. We considered shorter-term outcome measures those that capture functions that are more fluid and have the potential to change rapidly. For example, in the cognitive domain, working memory and fluency and some attentional aspects.

After determining the duration of outcomes for each measure, we graded tool quality $[11,13-15]$. Three criteria were used: (1) whether reliability and validity data was available in typically developing individuals and/or in neurodevelopmental disorders (ID, ASD, FXS, Rett syndrome), (2) whether the measure had been piloted in FXS clinical trials (Table 1; [4]), and (3) whether the measure addressed an important aspect of the FXS phenotype $[2,16]$. The grading was done using the COSMIN system that includes the following levels (from best to worst): strong, moderate, limited, unknown due to poor methodological quality, and no evidence available. Adapting these grades to our criteria, a strong ("+++") label required evidence of (1) compelling reliability and validity (e.g., excellent internal consistency) in typical individuals and/or 


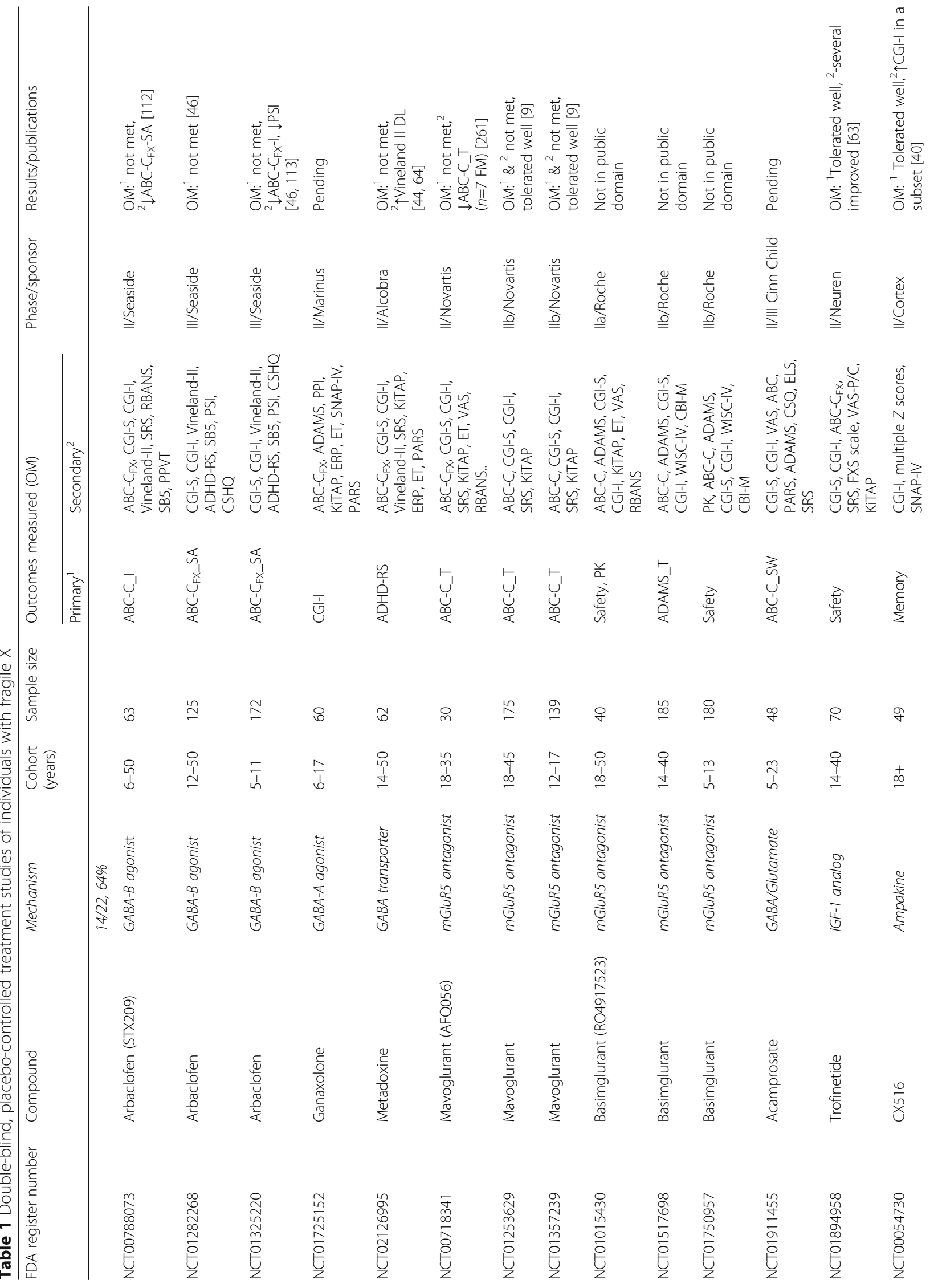




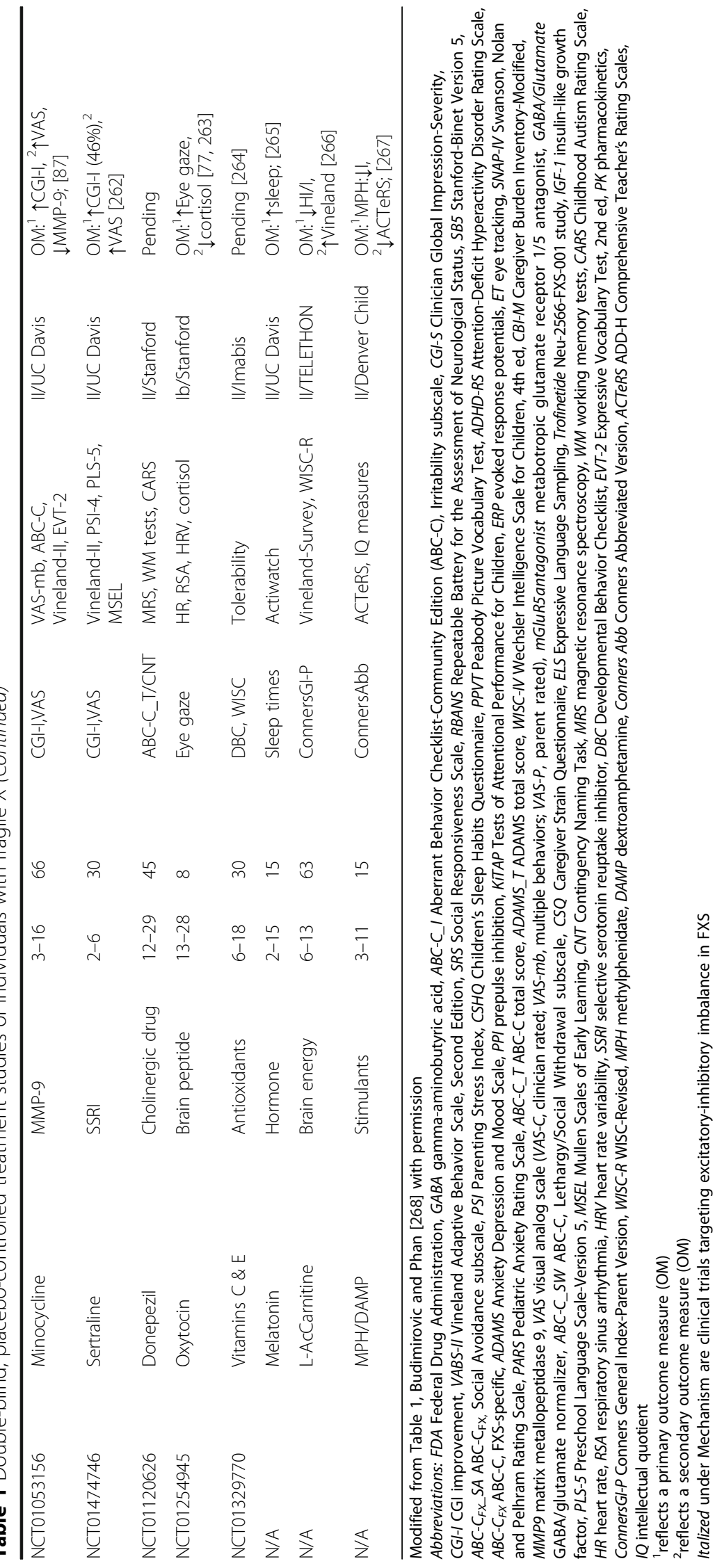


several neurodevelopmental disorders, including FXS, and (2) having been piloted in three or more FXS clinical trials that showed sensitivity to treatment. A moderate ("++") label required evidence of (1) acceptable reliability and validity data (e.g., good internal consistency) in typical individuals and/or one or more neurodevelopmental disorders, including FXS, and (2) being piloted in one or two FXS clinical trials. The limited ("+") label was considered if there was (1) weaker evidence of reliability and validity (e.g., fair internal consistency) in typical individuals and/or one neurodevelopmental disorders and (2) no experience in FXS clinical trials. The unknown due to poor methodological quality and no evidence available labels differed in that the former had some data on reliability or validity and/or the available data was of poor methodological quality.

In order to illustrate the psychometric properties we used for determining the tool quality of each instrument, here, we provide some quantitative parameters employed in psychometric assessments. Internal consistency, typically measured by the Cronbach's alpha coefficient, is evaluated by the following interpretative guidelines: excellent $(\geq 0.90)$, good $(0.80-0.89)$, fair $(0.70-0.79)$, and unacceptable $(<0.70)$. Multiple measures of reliability (e.g., test-retest, inter-rater) are usually evaluated by intraclass correlation coefficients as follows: excellent $(\geq 0.75)$, good $(0.60-0.74)$, fair $(0.40-0.59)$, and poor $(<0.40)$. For more information about psychometric evaluations, we recommend the reader our recent publication on anxiety measures in Rett syndrome [17] as well as the UK's NHS National Institute for Health Research comprehensive review of behavioral outcome measures for young children with idiopathic ASD [11]. Details about measurement properties of each instrument can be found in the key original publications, all included in the "References" section.

\section{Results}

The period between 2008 and 2015 saw tangible progress in controlled clinical trials in FXS, particularly those applying neurobiological-targeted treatments. Table 1 depicts studies in individuals with FXS that employed best practices methodology (randomized, double-blind, placebocontrolled trials). To date, a total of 22 such trials have been identified through search of literature and other sources; 19/22 (86\%) have been registered on the www.ClinicalTrials.gov website, and data on more than half of the studies have been published to date.

As expected from FXS's neurobiology, the vast majority of the studies have targeted a core excitatory/inhibitory imbalance in the disorder (italized under Mechanism, Table 1) primarily through either mGluR5 antagonists (mavoglurant-AFQ056, basimglurant-RO4917523) or GABA agonists (arbaclofen-GABA-B agonist, ganaxolone-GABA-A agonist, acamprosate-GABA-A/GABA-B agonist). These studies represent the majority of the total $(14 / 22,64 \%)$ and registered $(14 / 19,74 \%)$ trials. Reflecting that over two thirds of these trials were phase II, most of them have studied adults and adolescents (i.e., FDA recommendations indicate that novel drugs should be tested first in adults, particularly in vulnerable populations). Some trials targeted other glutamatergic components (AMPA receptors: ampakine CX-516) or more general synaptic mechanisms (IGF-1 tripeptide analog trofinetide, minocycline). The rest of the reported studies focused on other systems presumably affected by FMRP deficit: serotonergic and cholinergic drugs, the "social brain" neuropeptide oxytocin, compounds that affect metabolism, and other modulators.

Experience from these trials was used to re-assess the quality of outcome measures employed. Based on the latter data and the literature, the following sections reevaluate the utility and quality of available endpoints, grouped into the three categories used in the $2013 \mathrm{Re}$ port: Cognition, Behavior/Emotion, and Biomarker/ Medical. Evidence and recommendations for each area are outlined as follows: Background, Progress and plans in FXS, Potential measures, and Conclusions. Due to the large number and diversity of measures, the Behavior/ Emotion and Biomarker/Medical domains are divided into subdomains.

\section{Cognitive measures Background}

Individuals with FXS present with variable cognitive, adaptive, and language impairments. Boys with FXS who do not meet the criteria for ASD typically function in the mild to moderate ID range ( FSIQ 40-70), whereas those with ASD are often lower functioning [18, 19]. Expressive language is typically more affected than receptive language in individuals without ASD. Since cognitive impairments are core FXS phenotypes, it is postulated that most therapeutic interventions targeting key mechanisms related to FMRP deficit (e.g., mGluR5 antagonists, trofinetide) would have an impact on global or specific cognitive domains, including communication abilities [20, 21]. Most measures of cognition have been employed in observational studies, even after the beginning of the clinical trial era in FXS. The 2013 Report recommended (1) validation of an expressive language sampling technique to measure improvements in language described anecdotally by parents during clinical trials and (2) identification of measures of cognition and executive function that can be performed by individuals with FXS and for which floor effects can be avoided. The Working Group also recommended that, although promising measures were identified for several key domains, further data were needed on several properties 
before their use for clinical trials: (1) test-retest reliability, sensitivity, and validity; (2) validation over an expanded age range; and (3) feasibility and validity across the full range of affectedness. Here, we review measures of global cognitive function, including adaptive behavior skills, followed by instruments used for evaluating selective cognitive functions (Table 2).

\section{Progress and plans in FXS}

1. Global cognitive measures. These include tests of general intelligence and adaptive behavior skills.
The former yield IQ scores, including the Stanford-Binet Intelligence Scales (Fifth Edition, SB5) [22] and the Wechsler Intelligence Scales [23-25], and developmental scales that measure cognition in young children such as the Mullen Scales of Early Learning [26] and the Bayley Scales of Infant Development [27]. These instruments and adaptive behavior scales have been long used in FXS in the context of a variety of observational studies [28, 29], in particular the Vineland Adaptive Behavior Scales [30], now in its 3rd Edition (Vineland-3; [31]).

Table 2 Global and other cognitive domains and outcome measures in fragile $X$ syndrome

\begin{tabular}{|c|c|c|c|c|}
\hline Domain & Outcome measure & Shorter term & Longer term & Quality of tools \\
\hline \multirow[t]{2}{*}{ Intellectual quotient } & Wechsler_WAIS-IV, WPPSI-IV, WISC-V & & Yes & $++[23-25,32,33]$ \\
\hline & Stanford-Binet 5 & & Yes & $++[22,33,34,46,112]$ \\
\hline Multiple cognitive skills & NIH Toolbox Cognitive Battery & $Y_{e s}^{a}$ & Yes & $+[35-38]$ \\
\hline \multicolumn{5}{|l|}{ Adaptive behavior } \\
\hline & Vineland-II & & Yes & $++/+++[19,29,44,46-49,98,112]$ \\
\hline & ILS & & Yes & $+[97-99]$ \\
\hline & W-ADL & & Yes & $+[100]$ \\
\hline \multicolumn{5}{|l|}{ Memory \& Learning } \\
\hline & WJ working memory & Yes & Yes & $+[50,51]$ \\
\hline & RBANS & Yes & Yes & $+/++[53-56]$ \\
\hline & SB-5 Block Span & Yes & Yes & $+[22,33]$ \\
\hline \multicolumn{5}{|l|}{ Executive function } \\
\hline & KiTAP (4 subtests) & Yes & Yes & $++/+++[44,46,60-63,112]$ \\
\hline & WJ auditory attention & Yes & Yes & $+[50,51]$ \\
\hline & WJ digits reversed & Yes & Yes & $+[50,51]$ \\
\hline \multicolumn{5}{|l|}{ Language } \\
\hline & PPVT-4 & & Yes & $+[66,67,72-74]$ \\
\hline & EVT-2 & & Yes & $+[68]$ \\
\hline & CELF-5/P2 & & Yes & $+[66,69,70]$ \\
\hline & FMP & Yes & & $+[72-75]$ \\
\hline & ELS & Yes & Yes & $++[6,21,44,76]$ \\
\hline \multicolumn{5}{|l|}{ Social cognition } \\
\hline & SRS & Yes & Yes & $+/++[15,17,46,78,80,81,112,113]$ \\
\hline & Eye tracking & Yes & Yes & $++[85,87,88]$ \\
\hline & SPPA & $?$ & $?$ & $?[89]$ \\
\hline \multicolumn{5}{|l|}{ Academic achievement } \\
\hline & WJ Achievement III & & Yes & $?[90-93]$ \\
\hline & WIAT-II & & Yes & \\
\hline
\end{tabular}

Abbreviations: Wechsler_WAIS-IV Wechsler Adult Intelligence Scale, 4th ed, WPPSI-IV Wechsler Preschool and Primary Scale of Intelligence, 4th ed, WISC-V Wechsler Intelligence Scale for Children, 5th ed; Stanford Binet5, 5th edition, NIH National Institute of Health, Vineland-II Vineland Adaptive Behavior Scale-2nd Ed, ILS Independent Living Scales, W-ADL Waismen Activities of Daily Living Scale, WJ Woodcock Johnston, RBANS Repeatable Battery for the Assessment of Neurological Status, KiTAP Test of Attentional Performance for Children, PPVT-4 Peabody Picture Vocabulary Test-4th ed, EVT-2 Expressive Vocabulary Test-2nd ed, CELF-5/P2 Clinical Evaluation of Language Fundamentals-5th edition/Preschool-2nd ed, FMP fast-mapping performance, ELS expressive language sampling, SRS Social Responsive Scale, SPPA Self-Perception Profile for Adolescents, WIAT-II Wechsler Individual Achievement Test-II. Shorter term deemed suitable for clinical trials lasting $<12$ months; Longer term deemed suitable for clinical trials lasting $>=12$ months. Grading system: +++ strong evidence, ++ moderate evidence, + limited evidence, ? unknown due to poor methodological quality, blank cell no evidence available

${ }^{a}$ Yes applies for some subtests 
(a) General intelligence tests. While these cognitive measures have good psychometric properties, IQ tests have known floor effects when applied to individuals with significant cognitive impairment (i.e., below mild ID level), limiting their sensitivity to change, group and individual differences, and relative strengths and weaknesses within the individual. Hessl and colleagues have developed scoring modifications that address these shortcomings and allow better estimates of profiles of cognitive strength and weakness [32, 33]. The latest publication, which developed "deviation scores" for the SB-5 in individuals with FXS and idiopathic ASD with comorbid ID [33], suggests that the SB-5, with a very broad developmental range, may be an adequate longer-term outcome measure for tracking developmental progress in FXS trials. Altogether, the evidence of progress on the use of the SB-5 in FXS places it at the moderate tool quality level. As discussed in the adaptive behavior section, it is important to know the developmental trajectories of global cognitive measures. In general, IQ scores tend to decrease with age during childhood, reflecting a widening gap or slower progress in individuals with FXS compared to normal cognitive development. In fact, Quintin and colleagues, using the deviation method described above, showed in a group of 184 children and adolescents with FXS, aged 6-16 years, that the cognitive profile associated with FXS develops dynamically from childhood to adolescence and varies depending on cognitive domain [34]. Therefore, careful interpretation of changes of standard scores with age is needed, and the use of special scores that are not age-adjusted may be useful in detecting change in clinical trials (e.g., SB-5 changesensitive scores).

The NIH Toolbox Cognitive Battery (NIH-TCB) is a web-based and touch-screen response neuropsychological tool normed on children and adults [35], with a primary focus on its use as a series of cognitive endpoints such as executive function, attention, working memory, processing speed, episodic memory, single-word reading, and receptive vocabulary for a variety of populations and research questions [36]. It yields fluid reasoning, crystallized, and overall cognition composite standard scores analogous to the IQ scores described above. The battery is available in both English and Spanish, with recently demographically corrected normative standards for the English version [37]. The primary goal of the NIH-TCB is to employ a more efficient, common metric for cross-study comparisons, but it does not substitute in-depth, comprehensive neuropsychological batteries [36]. Piloting and validation of the NIHTCB in populations with ID (i.e., FXS, Down syndrome, and other forms of ID) to determine feasibility, reliability, validity, and sensitivity to change (after 2 years) are in progress as a four-site NICHD-funded project (1R01HD076189) awarded in response to the PAR-13-213, Outcome Measures for Use in Treatment Trials for Individuals with Intellectual and Developmental Disabilities (PIs: Hessl, Berry-Kravis, Riley, Gershon). A recently published series of pilot studies from this group using NIH-TCB measures provides preliminary evidence of feasibility, test-retest reliability, convergent validity, and sensitivity to group differences [38]. In theory, the NIH-TCB could be used as either a shorter- or longer-term outcome measures; we grade it as a limited quality tool until the complete study results with larger sample sizes become available and utility is also confirmed. More information about measurements of specific cognitive functions is provided below in sections 2-5.

(b) Adaptive behavior scales. This is a behavioral domain closely linked to cognition, representing to large extent the application of cognitive function to daily life. Thus, the Diagnostic and Statistical Manual for Mental Disorders $-5^{\text {th }}$ Edition (DSM-5)'s definition of ID places adaptive skills at the center of intellectual functioning [39]. Most recent data on adaptive skills in FXS has been obtained with the Vineland-II, which has been validated in children and adults with ID and used in populations such as ASD, FXS, Down syndrome, Angelman syndrome, and Rett syndrome [17, 40-43]. Despite this, the Working Group found that few adaptive behavior measures had been validated or standardized for populations with ID, and most had significant floor effects. The Vineland-II has been employed as a secondary outcome measure in three recent FXS clinical trials. In a randomized, double-blind, placebo-controlled, multi-site phase IIb trial of metadoxine (MDX, an ion pair salt of pyridoxine or vitamin B6 and L-pyroglutamate, Alcobra) in FXS (AL014 trial) (Table 1), involving 62 adolescents and adults, aged 14-50 years, Berry-Kravis reported that individuals with FXS gained an average of 2.6 standard score points on the Vineland-II Daily Living Skills domain following 6 weeks of treatment (vs. 1.4 standard score points in the placebo group; $p=0.035$ ) [44]. Additional analyses indicated that the effect was greater in younger individuals $(<18$ years) and 
those with higher, although not normal IQ. These results suggest that the Vineland-II Daily Living Skills domain may be sensitive to drug effects over a relatively short period of time. In two phase III trials of arbaclofen in males and females with FXS (STX209), a flexible dose trial involving 119 patients, aged $12-50$ years, and a fixed dose trial involving 159 patients, aged 5-11 years, no improvements were found on the Vineland-II Socialization. On the other hand, it was also reported that some patients derived benefit from arbaclofen on the Vineland-II Socialization subscale following a long-term open-label extension $[45,46]$.

Several recent studies have documented the extent to which standard scores on the Vineland-II may deviate relative to the normative data at particular age bands in FXS. For example, in a cross-sectional study of boys with FXS, standard scores on the Vineland-II declined between 1 and 4 points per year depending on the subdomain [47]. Similarly, in a large-scale longitudinal study involving 275 males and females with FXS, aged 2-18 years, Klaiman and colleagues reported that standard scores for males declined by approximately 3.3 points per year on average on the Vineland-II Composite, by 4.5 points per year on the Daily Living Skills domain, by 3.5 points per year on the Communication domain, and by 2.8 points per year on the Socialization domain [29]. Declines in standard scores with age were smaller in females and lessened as the subjects aged into adolescence. Interestingly, a recent longitudinal study involving 47 boys with FXS, between 9 and 15 years, reported that standard scores on the Vineland-II Socialization and Communication domains improved with age whereas scores on the Daily Living Skills remained relatively flat [48]. This indicates that the development of some adaptive behavior domains may be relatively less affected than others; however, more data are needed to clarify this age and gender differences.

Note: Decline in standard scores of cognitive or adaptive behavior measures with age reflects the fact that affected individuals are not keeping pace with the normative typically developing samples, rather than reflecting any loss of adaptive skills.

Altogether, these data indicate that the Vineland-II is a suitable instrument for longer-term studies (e.g., 1 year in length); however, these investigations will need to factor the natural decline or increase in scores at particular age bands. Strengths of the Vineland-II as a longer-term outcome measure include its low floor of 20 standardized points, ease of administration (parent interview), and relatively short administration time $(\sim 1 \mathrm{~h})$. Caveats of the measure include the potential for reporting bias, low sensitivity to change, and the fact that the psychometric properties of this instrument are unknown in the FXS population (although one study has reported that the internal consistency of the Vineland-II in individuals with ID is high [49]). At present, the Vineland-II should be considered a measure of moderate tool quality. Although no data are yet available, early application of the Vineland-3 [31] to FXS indicates a similar performance to the Vineland-II.

2. Memory and learning. The Working Group identified several memory tasks that mapped onto the FXS memory phenotype, such as the Auditory Working Memory and the Digits Reversed subtests of the Woodcock-Johnson III Tests of Cognitive Abilities $[50,51]$, the Corsi Blocks [52], and the SB-5 Block Tapping component of Visual Working Memory (visual-spatial sequential memory). While no published data are available on reliability or validity in subjects with FXS to determine their quality, these memory tasks have the potential of being used as either shorter- or longer-term outcome measures. The Working Group also proposed assessing longterm memory with the List Learning and Story Memory subtests of the Repeatable Battery for the Assessment of Cognitive Status (RBANS; [53, 54]). The RBANS is a neuropsychological assessment that covers five domains (Immediate Memory, Language, Attention, Visuospatial/Constructional, Delayed Memory), developed in part as a neuropsychological screening battery for adult patients with neurological disorders. The List Learning subtest showed significant improvement in an open-label trial of lithium in 15 individuals with FXS, aged 6-23 years $[54,55]$. A placebo-controlled trial of the ampakine CX516 in 49 patients with FXS, aged 18-49 years, did not find a significant improvement in their cognition based on composites of several subtests including primarily the RBANS; however, some of the RBANS subtests showed high feasibility and testretest reliability (List Learning, Story Memory, List Recognition, Digit Span) [40]. Administration of the RBANS via video teleconference remotely was found to be feasible and reliable when compared to face-to-face administration [56]. Thus, the RBANS could be used as either a shorter- or longer-term outcome measure, showing preliminary evidence of reliability and validity (and possibly sensitivity to change). This places the RBANS in the limited-moderate tool quality. 
3. Executive functioning (EF). There is compelling evidence that deficits in $\mathrm{EF}$, present in most neurodevelopmental disorders, are highly characteristic of individuals with FXS $[57,58]$ with performance often below mental age expectations [59]. The Working Group identified several measures of EF that appear to be well suited for use in clinical trials (i.e., KiTAP 4 subtests, Woodcock-Johnson Rapid Naming). Nonetheless, some of these measures may be beyond the ability of lower-functioning individuals.

(a) KiTAP. This is a set of computer-based assessments of EF, which was standardized for typically developing children, ages 6-10 years [60]. The KiTAP is composed of eight tests that vary in length and difficulty level, capturing attention, inhibitory control, and cognitive flexibility [61]. An advantage of the KiTAP for FXS is the visual nature of the tasks, which taps into a relative strength. A pilot validation study of the KiTAP in FXS, including 36 males and females, aged 750 years, identified four subtests that generate measures of EF with good feasibility, reproducibility, and minimal ceiling and floor effects [62]. These four specific subtests were Alertness reaction time, Distractibility commission errors, Go/ No-Go commission errors, and Flexibility errors, all of which demonstrated good clinical validity [i.e., correlation with Attention or Hyperactivity subscales of the Aberrant Behavior ChecklistCommunity (ABC-C) or the Behavioral Assessment for Children Scale (BASC)] and test-retest reliability [62]. All these features led to the inclusion of the KiTAP in five clinical trials. The KiTAP could be used as a shorter-term outcome measure, with psychometric evidence supporting a moderate to strong tool quality label, as we wait more data on sensitivity to treatment (i.e., [63], Table 1). In the recent phase II trial of metadoxine mentioned in the previous section on adaptive behavior, scores on the KiTAP Go/No-Go subscale improved significantly with a relatively large effect size [44, 64]. EF measures in the NIH-TCB are currently under investigation.

The Working Group also suggested the use of informant report measures (e.g., the Behavior Rating Inventory of Executive Function or BRIEF [65]), which may prove useful when direct assessment is not possible. However, a recent pilot study aiming to validate the NIH Toolbox Cognitive Battery for ID, including those with FXS, showed weak correlations between caregiverreported EF on the BRIEF and the objectively measured similar constructs of EF (attention, inhibitory control, and cognitive flexibility) on the $\mathrm{NIH}$
Toolbox battery (see Hessl et al. [38]). The lack of association could indicate inadequate validity of the BRIEF for this population or discrepancy between laboratory-based assessment and real-life EF. Therefore, further psychometric work on the application of the BRIEF to FXS and other ID groups is important prior to recommending its use in clinical trials.

4. Language measures.

(a) Standardized tests. There are numerous standardized language tests that have proven useful in describing the profile of impairments characteristic of individuals with FXS [66]. These include the Peabody Picture Vocabulary TestFourth Edition (PPVT-4; [67]), for measuring receptive vocabulary, and the co-normed Expressive Vocabulary Test-Second Edition (EVT-2; [68]). The PPVT-4 and EVT-2 are direct, individually administered measures that are suitable for a wide range of age and ability. They provide growth scale values based on Rasch scaling, which would be useful for tracking intra-individual change. Other standardized tests that have been used to characterize language function in individuals with FXS include the Clinical Evaluation of Language Fundamentals-Fifth Edition (CELF-5) and CELF Preschool-Second Edition (CELF-P2) [69, 70], which have subtests for various language components, including expressive syntax, a problem area for individuals with FXS [66]. Despite their wide application in FXS and ID in general, none of these standardized tests have been formally evaluated in these populations in terms of psychometric properties of relevance for clinical trials. Pending more research, these standardized language tests could serve as longer-term outcome measures as they tap into more crystallized type of knowledge. Evaluation of measurement properties places them in the no evidence available category.

(b) Fast-mapping measures. In contrast to standardized language tests, which largely measure the accumulated products of language learning, there have been recent attempts to create measures that index language learning in real time, an approach that is especially promising for shorter-term clinical trials. The best studied of these "process" measures for FXS are fast-mapping tasks. Fast-mapping is an associative learning process in which children form an initial representation of a word's meaning by inferring a correspondence between a novel label and the speaker's intended referent [71]. Fast-mapping tasks usually are limited to the earliest phases of learning a word. Several recent studies have demonstrated that fast-mapping tasks can be completed by 
minimally verbal males with FXS, as young as age 4 years [72]. These tasks also document age-related deficits in learning that distinguish males with FXS from similarly aged, cognitively matched males with nonsyndromic ASD [72-74]. Although most fastmapping tasks are administered by an examiner in a face-to-face setting, computer-administered versions have been developed [75], increasing the feasibility of the task for clinical trials. Evidence of validity has been provided by studies documenting correlation with standardized test of vocabulary, such as the PPVT-4 [72-74]. Despite this, the type of psychometric studies needed to establish the appropriateness of fast-mapping tasks for clinical trials for FXS has yet to done, including evaluations of test-retest reliability and sensitivity to change. Thus, in terms of measurement properties, fast-mapping measures fall into the no evidence available category.

(c) Expressive language sampling (ELS). In contrast to the forgoing measures, psychometric work has been conducted on ELS and the Working Group concluded that these procedures held the most promise for immediate use in clinical trials, provided that the samples are collected in contexts that are sufficiently structured to ensure consistency of the interaction across participants and occasions of measurement. Dyadic conversation with an examiner and narrative retelling have been the most widely used ELS procedures with individuals with FXS [76]. A pilot validation of ELS in FXS, including 36 males and females, aged 5-36 years, showed very good reproducibility and clinical validity and led to its use in three clinical trials [20]. Results of ELS assessments from these clinical trials have not yet been reported. A NICHD R01 project (HD074346; PIs: Abbeduto, Berry-Kravis, Sherman, Edgin, Sterling) was funded to perform optimization and validation of the ELS in FXS and Down syndrome across five sites and also to measure its response to change after 2 years. Pending the results of this work, we can conclude that ELS procedures could be used as either shorter- or longer-term outcome measures. Existing evidence supports a moderate tool quality label, although it should be noted that transcription of samples can be highly timeconsuming which may make it difficult to use in very large trials unless increased automation of transcription can be developed or sample length can be reduced without loss of validity.

5. Social cognition. The Working Group stated that measures of social cognition have not been well studied in FXS. It was noted that the relationship between performance on such measures and actual social behavior is not strong [77]. The Working Group concluded that both the Social Responsiveness Scale (SRS) [78] and the use of eye-tracking technology to assess attention to social events were promising but required further validation in FXS.

(a) Social Responsiveness Scale (SRS). This is a 65-item parent/caregiver/teacher rating scale used to assess children on five subscales: Social Awareness, Social Cognition, Social Communication, Social Motivation, and Autistic Mannerisms (Restricted Interests and Repetitive Behavior in SRS-2) [79, 80]. The second edition of the SRS (SRS-2; [78]) also contains a Preschool Form. The SRS-2 can be used as a screener in clinical or educational settings, an aid to clinical diagnosis, or a measure of response to intervention, but questions remain about its usefulness as an outcome measure sensitive to change in clinical trials in part because the scale's criterion validity is lower than the ADI-R's [15]. The SRS's psychometric properties are rather robust. Factor analyses support a one-factor solution in populations at risk for ASD, limiting the SRS's use to an overall assessment of social responsiveness [78]. The SRS's internal consistency [80-82] and test-retest reliability [8183], including parents-teachers [83, 84] and motherfather [81] correlations, indicate reasonable psychometric properties (e.g., good to excellent test-retest reliability). In addition, published data support good convergent and discriminant validity of the SRS with the Child Behavior Checklist (CBCL) $[81,82]$. The SRS seems suited for studies evaluating shorter- or longer-term effects of interventions. Because the SRS has only been applied to a single openlabel trial in FXS, we labeled it as a limitedmoderate tool. Additional information is provided in the section "Autistic behavior", under the "Behavior and Emotion measures" section.

(b)Eye tracking/pupillometry. Eye-gaze avoidance is a major feature of FXS [2, 3]. A pilot study of social gaze behavior in FXS quantified gaze avoidance and pupillary reactivity to emotional faces, including 15 males and females, aged 7-51 years, revealing good feasibility and excellent testretest reliability [85]. A customized eye-tracking configuration was used to quantify social gaze while engaging in a naturalistic face-to-face social interaction with a female experimenter as opposed to static faces used in prior protocols [77]. Participants with FXS spent significantly less time looking at the examiner's face and had shorter episodes of social gaze than controls. It was proposed that this paradigm could be employed in clinical trials to provide a more naturalistic measure of social gaze than protocols using images of faces $[77,86]$. To 
date, the abovementioned eye-tracking protocol [85] has been used in several clinical trials, with preliminary evidence of pupillometry's sensitivity to treatment in a minocycline trial [87] as well as improvement in social gaze in a mavoglurant trial [88]. Data from other clinical trials are awaiting analysis for evidence of sensitivity to change. Then, eye tracking/pupillometry in FXS could be used as a shorter-term outcome measure with evidence for moderate to strong tool quality. Although eyetracking protocols can be easily standardized across sites, one potential limitation is the cost of eyetracking equipment that may limit its utility in smaller trials.

(c) Self-Perception Profile for Adolescents (SPPA). The application of the SPPA, which elicits self-ratings of social relationships, friendships, and self-worth, among other domains, in recent social cognition studies revealed significant differences between adolescent females with FXS and typical agematched peers [89]. Moreover, scores on the SPPA correlate with other social-cognitive measures, such as "reading" thoughts and feelings from a photograph of a face [90]. Parent reports and the SPPA were discrepant, with parents rating adolescents with FXS as more impaired than the adolescents rated themselves, which is a common finding among several populations. Awareness of the discrepancy between self and parent perceptions of social functioning is important for determining the need for intervention and its assessment. At present, although a promising measure, evidence for the use of the SPPA in clinical trials is lacking (i.e., no evidence available category).

6. Academic achievement. The Working Group recommended the use of academic achievement measures as indicators of longer-term outcomes. The expectation is that changes in language, memory, and EF are likely to lead to changes in the academic domain. In order to better understand the academic domain in FXS, language skills, reading ability, and phonological awareness have been studied in affected boys. As in typically developing boys, reading skills are also significantly correlated with delayed phonological awareness and level of non-verbal cognition in boys with FXS [90]. Young males with FXS display reading abilities that are commensurate with their cognitive expectations; however, their phonological skills are weaker than expected [91]. In conclusion, evidence is lacking on academic achievement and related measures for determining their usefulness and tool quality in FXS clinical trials (i.e., no evidence available category).

7. Learning process measures. Process measures may also be more sensitive to shorter-term drug effects than "product" measures, which are essentially the result of a learning process and, therefore, "accumulate" gradually to measurable levels of relevance to daily functioning and quality of life [92]. For example, in a recent study employing a "process" measure of change, Hall and colleagues [93] examined the rate at which lower-functioning boys with FXS, aged 1023 years, were able to learn the relationships between mathematical stimuli after 2 days of training presented either on a computer or by a therapist [92]. By tracking learning on a trial-by-trial basis, these authors found that boys with FXS were significantly slower to learn to match fractions to pie charts in comparison to age- and IQ-matched controls with ID [92]. These data suggest that meaningful differences in the rate at which boys with FXS learn new material could be detected in this population. Studies expanding on these findings are also needed to determine the usefulness of "process" measures for clinical trials in FXS.

\section{Potential measures}

1. Learning paradigms. This type of measures, with dynamic and regular training over time, may have excellent potential for use as cognitive endpoints in FXS clinical trials. In contrast to traditional outcome measures, which are evaluated at several key points during a trial, learning paradigms can provide data on progress much more often, providing a detailed "slope" of progress or change. Moreover, learning paradigms might more closely match progress in academic achievement or acquisition of skills as they occur in the individual's environment rather than laboratory-based measures. In a sense, learning paradigms reflect a non-pharmacological intervention, which may progress more rapidly in clinical trials due to synergistic effects with a targeted pharmacological treatment. The ongoing Cogmed Working Memory Training FXS clinical trial (PI: Hessl), supported by the John Merck Fund, provides an example of how daily cognitive training can be tracked on a trial-by-trial basis, yielding a high-resolution slope of change. Results of this double-blind placebo-controlled study should be available in 2017; however, open-label results of a FXS Cogmed feasibility study involving 17 males and females, mean age 12.49 years, were reported by $\mathrm{Au}$ and colleagues [94].

Learning paradigms focused on language have recently been shown to have efficacy for individuals with FXS over a wide age range (e.g., $[95,96])$ and, like Cogmed, could be useful as outcome measures, with the added benefit of "boosting" the effects of pharmacological treatments. 
2. Novel adaptive behavior measures.

(a) Independent Living Scales (ILS; [97]). The ILS is a measure of adaptive behavior that assesses the ability to function independently and handle reallife situation. It was originally designed to evaluate adaptive behavior in aging populations but has recently been employed to evaluate adaptive skills in individuals with FXS [98]. The ILS has five subdomains, two composite factors, and a full-scale standard score ranging from 55 to 115 . Psychometric properties, such as test-retest stability and internal consistency, have been reported to be strong [97] but they have not been investigated in detail in FXS. In a recent study mentioned above [99], 70 individuals with FXS (males and females), aged 15-25 years, showed comparable ILS domains and factors to those of age/IQmatched individuals with ID [98]. The authors used raw scores to conduct the comparison because $77 \%$ of males and $17 \%$ of females with FXS were found to have scores at a floor level for the test [98]. Therefore, the ILS appears to have limited utility as a directly administered longer-term outcome measure of adaptive behavior for males with FXS, and another recent study shows that it is also promising for use in affected females [99].

(b)Waisman Activities of Daily Living (W-ADL) Scale. Another measure with potential for adolescents and adults with developmental disabilities is the W-ADL, which has been recently tested in 1014 individuals including 147 with FXS [100]. The W-ADL covers 17 daily living activities, and it has shown excellent psychometric properties, including internal consistency, criterion and construct validity (e.g., correlated with Vineland scales screener), and no floor or ceiling effects. Importantly, W-ADL scores differentiate maternally reported level of ID (mild, moderate, severe, profound) [100]. A recent application of the W-ADL to 147 adolescents and adults with FXS, aged 12-48 years, showed an improvement in adaptive skills over time [101]. While the W-ADL is recommended for surveys and epidemiological research, it may also be useful as an outcome measure for clinical trials.

(c) Scales of Independent Behaviors-Revised (SIB-R; [102]). While only applied as a measure of maladaptive behavior, the SIB- $R$ is mainly an adaptive behavior instrument that has not been evaluated systematically in FXS.

\section{Conclusions}

Most of the conclusions of the Working Groups continue to be valid. As the 2013 Report indicated, there is only sparse evidence on reliability and validity for most of the instruments used to measure cognitive deficits in FXS. However, several pilot projects with high potential cognitive measures (KiTAP, ELS, NIH-TCB) have been completed since the 2013 Report and larger validation studies are in preparation or ongoing. Thus, new data in the Cognition domain may lead to stronger recommendations for some tools in the next few years. In terms of adaptive behavior, an area with a long track record of observational studies in FXS, new measures including the ILS, and the $\mathrm{W}-\mathrm{ADL}$, are promising but need to be formally assessed. It is expected that the recently released Vineland-3 will perform similarly to the Vineland-II in FXS, although will be more relevant to current adaptive skills (e.g., electronic device use). Studies to evaluate the Vineland-3 in FXS are underway. We conclude that the overall evidence for cognition-related outcome measures in FXS places them in the limited to moderate quality range, with most instruments adequate for assessment of longer-term changes (i.e., some evaluating specific cognitive functions may be appropriate as shorter-term endpoints).

\section{Behavior and Emotion measures}

This section covers some of the most distinctive phenotypical features of FXS, which are a major focus of current clinical management [2]. The range of behavioral abnormalities in FXS is wide, with five areas commonly recognized: maladaptive/disruptive behaviors, ADHD-like behaviors, stereotypic and repetitive behaviors, anxiety, and autistic features [3]. In addition to the frequent combination of anxiety and autistic features with other problematic behaviors, hypersensitivity or over-reactivity (hyperarousal) to stimuli complicates the delineation of abnormal behaviors in children with FXS [3]. Other general issues that deserve consideration include the fact that (1) most behavioral measures have been developed for populations with relatively normal cognitive function, (2) without the specific goal of detecting change over time, and that (3) the conceptual framework/construct underlying specific abnormal behaviors is not fully developed in FXS, and (4) may differ from the one applied to the general population (e.g., anxiety in intellectual disabled groups such as FXS). The latter points have also been raised in the reviews of outcome measures in ASD [10-15]. Here, we review measures of abnormal Behavior and Emotion divided into the aforementioned five areas.

In 2013, the Working Group on Behavior and Emotion made the following general recommendations regarding outcome measures targeting the behavioral domain: (1) to determine the psychometric properties, namely reliability, validity, and sensitivity (including sensitivity to change), of several currently available behavioral measures within the FXS population; (2) to establish the specificity of the measured constructs to FXS by examination of correlations between such measures and valid FXS biomarkers (e.g., brain imaging) and gene-dose (e.g., FMRP); 
(3) to consider the development of a new behavior rating scale for FXS to cover the phenotype and the full range of associated symptoms; and (4) to supplement traditional psychometric studies with data from focus groups that include patients or their proxies and other caregivers to provide input on the construct validity, interpretability, and feasibility of measures of interest. We will review these general recommendations for the Behavior/Emotion domain in each specific section (subdomain).

Table 3 depicts behavioral and emotion outcome measures, their suitability for quantifying shorter- vs. longer-term effects in clinical trials and the quality of their measurement properties. In general terms, because of the dynamic nature of problematic behaviors, their measures tend to reflect shorter-term effects. However, because many instruments can be applied repeatedly without a training effect, behavioral endpoints could also be used for evaluating longer-term outcomes. The field is dominated by informant-based rating scales using Likert type of scoring, with variable quality of measurement properties. An additional discussion of these issues is provided in the following "Problem behaviors: focus on disruptive behavior domain" section.

\section{Problem behaviors: focus on disruptive behavior domain Background}

Maladaptive behaviors, in particular externalizing behaviors, termed here "problem behaviors", are one of the major clinical concerns with functional and quality-oflife implications in FXS $[3,103]$. Therefore, there is a greater experience in this behavioral area than in others. Measures previously used in clinical trials in FXS cover a wide range of problem behaviors, including ADHDlike behaviors (e.g., hyperactivity), stereotypic and repetitive behaviors, and anxiety. Because of the large volume of information on each of these types of behaviors, they will be reviewed in separate sections (i.e., subdomains). In this initial section, we will focus on irritability/agitation/aggressive behavior, termed here disruptive behavior, and self-injurious behavior. As stated in the 2013 Report, problem behaviors are typically evaluated by informant-based rating scales, which are completed by a

Table 3 Outcome measures of problem behaviors: focus on disruptive behaviors domain in fragile $X$ syndrome

\begin{tabular}{|c|c|c|c|c|}
\hline Subdomain & Outcome measure & Shorter term & Longer term & Quality of tools \\
\hline \multicolumn{5}{|l|}{ Irritability/aggression } \\
\hline & $A B C-C_{-} I$ & Yes & Yes & $+++[54,55,104-110]$ \\
\hline & $A B C-C F X \_I$ & Yes & Yes & $+++[9,46,103,111-113]$ \\
\hline Stereotype/self-injury/aggression & BPI-S & Yes & & $+[115-117]$ \\
\hline Variety of behaviors & FXS Rating Scale & Yes & Yes & $+[63,114]$ \\
\hline Inattention & Conners/ADHD-RS & Yes & & $++[44,64,120,122,126]$ \\
\hline Hyperactivity/impulsivity & Conners/ADHD-RS & Yes & & $++[44,64,120,122,126]$ \\
\hline Hyperactivity/impulsivity/inattention & CBCL_ADHD Scale & Yes & & $++[130,131]$ \\
\hline Hyperactivity/impulsivity/inattention ${ }^{a}$ & SNAP-IV & Yes & & $++[40,113,267]$ \\
\hline \multicolumn{5}{|l|}{ Anxiety } \\
\hline Social withdrawal/anxiety & ABC-C_SW & Yes & Yes & $+++[9,19,28,46,103,111-113]$ \\
\hline \multicolumn{5}{|l|}{ Social anxiety } \\
\hline & PARS & Yes & Yes & $++[134,138]$ \\
\hline & ADAMS & Yes & Yes & $++[13,17,113,132,138]$ \\
\hline & ADAMS Social Avoidance & Yes & Yes & $++[17,139]$ \\
\hline & CBCL Withdrawn & Yes & Yes & $+[18,19,135]$ \\
\hline & CBCL's DSM-Anxiety & Yes & & $+[136,137]$ \\
\hline & SCARED & Yes & & $+[10,13]$ \\
\hline Repulsive/compulsive behavior & RBS-R & Yes & & $+[15,179-184]$ \\
\hline Social reciprocity/avoidance & SRS & Yes & Yes & $+/++[15,46,78-81,112,113]$ \\
\hline
\end{tabular}

Abbreviations: $A B C-C$ Aberrant Behavior Checklist-Community Edition, $A B C-C_{-} / A B C-C$, Irritability subscale, $A B C-C_{F X} / A B C-C$, FXS-specific, Irritability subscale, $B P I$ Behavior Problems Inventory, ADHD-RS Attention-Deficit Hyperactivity Rating Scale, CBCL_ADHD Scale Child Behavior Checklist_ADHD subscale, SNAP-IV Swanson Nolan and Pelham Questionnaire-4th ed. ABC-C_SW ABC-C, Lethargy/Social Withdrawal subscale, PARS Pediatric Anxiety Rating Scale, ADAMS Anxiety Depression and Mood Scale, CBCL Withdrawn CBCL Withdrawn Subscale, CBCL's DSM-Anxiety CBCL's Diagnostic and Statistical Manual-Anxiety Subscale, SCARED Screen For Child Anxiety Related Emotional Disorders, RBS-R Repetitive Behavior Scale-Revised, SRS Social Responsive Scale, shorter term deemed suitable for clinical trials lasting $<12$ months; longer term deemed suitable for clinical trials lasting $>=12$ months. Grading system: +++ strong, ++ moderate, + limited evidence, ? unknown/poor methodological quality, blank cell no evidence available

${ }^{a}$ Oppositional and defiant items are also included 
parent, caregiver, or clinician. We continue to consider this a reasonable approach because of their ease of use, cost, and application to multi-site studies. However, it is important to point out that these measures involve evaluations of behavioral abnormalities during the last few weeks prior to rating and represent average estimates of a behavioral "style" rather than a dynamic process. By far, the best characterized instrument is the Aberrant Behavior Checklist-Community (ABC-C) [104]. The $\mathrm{ABC}-\mathrm{C}$ covers five categories of problem behaviors, but it is limited in its evaluation of ADHD-, anxiety-, and autistic-like behaviors. As depicted in Table 3, the ABC$\mathrm{C}$ has been widely applied to trials involving individuals with ID or ASD [104-106]. The 2013 Report summarized several limitations of the ABC-C (e.g., test-retest reliability) that can affect its sensitivity to change and, therefore, its ability to detect response to treatment. Despite this, the ABC-C has generally good psychometric properties and a successful track record for documenting improvements in disruptive behavior in controlled trials of idiopathic ASD [107, 108] and openlabel trials of lithium, aripiprazole, and donepezil in children and adults with FXS $[55,109,110]$ (Table 3). As other measures that were developed for ID or ASD, the relevance of the ABC-C to the FXS behavioral phenotype characterized by prominent anxiety-like behaviors was questioned. Therefore, the Working Group decided to revise and validate the ABC-C for FXS and, after improving its psychometric properties, to determine its sensitivity in clinical trials.

\section{Progress and plans in FXS}

(1)Aberrant Behavior Checklist-Community (ABC-C), adapted for FXS (ABC-C $\left.C_{F X}\right)$. The process of refactoring the $\mathrm{ABC}-\mathrm{C}$ for FXS, through a multi-site Fragile $\mathrm{X}$ Clinical and Research Consortium (FXCRC) collaboration [111], led to the elimination of three items and, more importantly, to a different factor structure that added novel subscales that seem to better represent autistic and social anxiety behaviors. The $A B C-C_{F X}$ factor structure was further supported by a subsequent study conducted by Novartis and RTI/Our Fragile X World [103]. The ABC-C has been used in every drug trial performed in FXS since its publication in late 2011, including a pediatric arbaclofen trial that showed some significant improvements [112, 113]. Based on these controlled trials and their open-label extensions, it has been concluded that the $\mathrm{ABC}-\mathrm{C}_{\mathrm{FX}}$ could be used as either a shorter- or longer-term outcome measure (see Table 3). Considering its initial psychometric evaluation [111], re-assessment [103], and application to FXS trials, in which several subscales have demonstrated sensitivity, the $\mathrm{ABC}-\mathrm{C}_{\mathrm{FX}}$ falls into the moderate-strong category of instruments (two positive randomized placebo-controlled trials: phase 2 mavoglurant [9], phase 3 pediatric arbaclofen [46, 113]). This labeling corresponds mainly to the ABC$\mathrm{C}_{\mathrm{FX}}$ Irritability subscale, pertinent to the Disruptive Behavior domain. However, two other $\mathrm{ABC}-\mathrm{C}_{\mathrm{FX}}$ subscales, specifically Social Avoidance and Hyperactivity, demonstrate many of the tool quality features of the Irritability subscale in a phase 3 pediatric arbaclofen study [113]. The main criticism continues to be its limited coverage of some key areas (e.g., non-social anxiety), which is discussed below, as well as the susceptibility to placebo effects. Nonetheless, rating variability and placebo effects are not specific to the $\mathrm{ABC}-\mathrm{C}_{\mathrm{FX}}$ but are associated with any parent/caregiver report measure.

(2)Fragile X Syndrome Rating Scale (FXSRS). As concluded by the Working Group, development of a new behavior rating scale covering the FXS behavioral phenotype and associated symptoms is a worthwhile effort. An initial attempt has been completed in the context of a trofinetide phase II trial conducted in adolescents and adults with FXS by Neuren Pharmaceuticals $[63,114]$. The FXSRS is a 34-item rating scale that covers a wide range of behavioral symptoms, including a FXS Core Phenotype subscale (i.e., 10 symptoms more prominent or prevalent in FXS than in other neurodevelopmental disorders), a FXS with Autism Phenotype subscale (i.e., six features shared to a similar degree by FXS and idiopathic ASD), and an Associated Phenotypic Features subscale (i.e., 18 features shared to a similar degree by FXS and general ID) subscales [114]. Items are answered using a 4-point severity or frequency scoring system. Publication of the FXSRS is pending; however, description of features in a group of 70 males with FXS, aged 12-45 years, presented at a meeting in 2014 [114], suggests it meets the requirements stated in the 2013 Report, including its potential use as shorter- and longer-term outcome measure (Table 3). Although grading of the FXSRS at this point is not possible or limited, it was employed in the aforementioned trofinetide study. This trial was reported as positive, based on an analytical strategy using multiple endpoints including the FXSRS (Berry-Kravis et al. 2016; www.neurenpharma.com/IRM/PDF/1557/ TrofinetidesuccessfulinPhase2trialinFragileX).

(3)Behavior Problems Inventory-Short Form (BPI-S; [115]). The BPI-S is a 30-item parent report measure that includes aggressive/destructive, self-injury, and stereotypic behaviors subscales rated over the previous 2-month period. Items are answered using a 5point frequency and a 4-point severity scoring system. Studies employing an earlier version of the 
scale (BPI-01; $[116,117])$ reported that in 50 males with FXS, aged $8-24$ years, $79 \%$ engaged in selfinjurious behavior (SIB), $98 \%$ in stereotypic behavior, and $75 \%$ in aggressive/destructive behavior. Moreover, $33 \%$ of the sample demonstrated aggression and $80 \%$ stereotypic behavior, both on a daily basis. The BPI-S could be used as either shorter- or longerterm outcome measure. However, it has not been subjected to reproducibility analyses in FXS and, at present, it is a limited quality tool.

\section{Potential measures}

A review of the ID and ASD literature supports the need for behavioral measures with better psychometric properties, particularly those relevant to detecting response to interventions. The recent National Institute for Health Research's review of behavioral outcome measures in ASD, mentioned in the "Material and methods" [11], concluded that there was "patchy evidence on reliability and validity". Of the six measures under evaluation, only two had acceptable properties [12]: the Child Behavior Checklist (CBCL) and the Home Situations QuestionnairePervasive Developmental Disorders version (HSQ-PDD). As also suggested by observational studies in FXS $[18,19]$, CBCL's main limitation is the lack of evidence on content validity for use with individuals with ID or ASD, particularly for those below the mild ID level. On the other hand, the HSQ-PDD has potentially relevant items but it is a relatively newer measure still under development. Thus, the CBCL and HSQ-PDD are instruments with potential as supportive outcome measures, but not as primary endpoints in intervention studies in FXS.

\section{Conclusions}

The $\mathrm{ABC}-\mathrm{C}_{\mathrm{FX}}$ is an adequate instrument for disruptive behavior in FXS, with measurement properties at the moderate-strong quality level, with the $\mathrm{ABC}-\mathrm{C}_{\mathrm{FX}}$ Irritability subscale demonstrating sensitivity in two FXS trials $[112,113]$ which could be used as either a shorteror longer-term outcome measure. Despite the strengths of the $\mathrm{ABC}-\mathrm{C}_{\mathrm{FX}}$, the fact that some key problem behaviors are not represented and that many items are not disorder-specific, the recommendation in the 2013 Report to develop a more comprehensive and FXS-specific outcome measure is still valid. The FXSRS could meet this apparent need; however, data are not yet available for a complete evaluation of this novel instrument.

\section{Attention-deficit hyperactivity disorder (ADHD)-like behaviors \\ Background}

Characterized by hyperactivity-impulsivity and/or inattention symptoms (DSM-5) [39], ADHD is one of the most common and potentially impairing behavioral issues in individuals with FXS. Evidence of its importance comes from family surveys [103, 118], several observational studies (reviewed in [3]), and a recent clinical study reporting that school-age children with FXS were significantly more likely to receive a professional diagnosis of ADHD compared to similar aged children with either Prader-Willi syndrome, Williams syndrome, or velocardiofacial syndrome [119].

\section{Progress and plans in FXS}

A limited number of recent clinical trials in FXS have included measures of ADHD as primary or secondary endpoints (Table 1), in addition to the $A B C-C_{F X}$ (see the "Problem behaviors: focus on disruptive behavior domain" section) that, as mentioned in the preceding section, includes a 16-item Hyperactivity subscale with many of the features of the strong Irritability subscale $[103,111]$.

ADHD Rating Scale-IV (ADHD-RS-IV; [120]). In the previously mentioned randomized phase II trial of metadoxine in FXS (AL014 trial), Berry-Kravis and colleagues (2015) found no significant reduction over placebo in scores on the Inattentive subscale of ADHD-RS-IV [120] and no differences between drug and placebo on the total score of the ADHD-RS-IV (employed as a secondary outcome measure) [44]. The ADHD-RS-IV contains 18 items that correspond directly to the symptoms of ADHD [120] on the Diagnostic and Statistical Manual for Mental Disorders-4th Edition (DSM-IV) [121] and the most recent DSM-5 version [39]. A clinicianadministered version of the scale is also available [122], which was used with some adaptations for ID in the MDX trial [122]. Although the psychometric properties of the ADHD-RS-IV have been reported to be good, there are no normative data available for this scale in individuals with FXS, and many of the items are difficult to interpret in FXS due to confounding ID. The scale could be used for measuring shorter-term effects, but it has limited tool quality due to the problems with content and interpretation in FXS.

\section{Potential measures}

Four potential measures of ADHD that have been employed in recent studies in FXS [123-126] are summarized below.

(1) The ADHD Test (ADHDT; [123]). A study employing the ADHDT reported that 68 males and females with FXS, aged 15-25 years, displayed a good range of scores [125]. The ADHDT contains 36 items divided into 3 subscales: Hyperactivity (13 items), Impulsivity (10 items), and Inattention (12 items). The ADHDT was normed to a group of 3- to 23-year-old individuals who met the DSM-IV criteria for ADHD. The psychometric properties of the ADHDT have been 
reported to be good, but its only application to FXS is as a measure of ADHD symptoms in conjunction with the $\mathrm{ABC}-\mathrm{C}_{\mathrm{FX}}$ Hyperactivity subscale.

(2) Conners Rating Scales-Revised [124]. Another recent study involving 46 boys with FXS, aged 4-11 years, showed "stable and striking impairments in inattention" on the Conners Teacher Rating Scale (CTRS) and the Conners Parent Rating Scale (CPRS) [126]. Both scales have four subscales: ADHD Index, Hyperactivity, Cognitive, and Oppositional, scored on a 4point Likert scale, and an age-normed $T$ score above 70 on the ADHD index is indicative of an ADHD diagnosis. The CPRS has reported good to excellent internal consistency across the four scales [124, 126], with scores on the CPRS generally higher than on the CTRS.

(3) Child Behavior Checklist (CBCL)'s ADHD subscale [127]. Several observational studies have employed the CBCL (ages 1.5-5 years, ages 6-18 years; [127, 128]) as a measure of ADHD symptoms in FXS [reviewed in [3]. The CBCL is a 118 -item parent rating scale used to assess internalizing and externalizing symptoms in children without ID. Items are answered using a 3-point Likert scale. The DSM-ADHD scale is one of several DSM scales of the CBCL based on criteria from the DSM-IV, with $T$ scores above 70 being indicative of an ADHD diagnosis. The CBCL was normed on a large, representative sample of children and adolescents and, in this population as well as in idiopathic ASD, has overall good psychometric properties [11, 129]. In a recent longitudinal study employing this measure, Grefer and colleagues reported that the mean DSM-ADHD raw scores increased over a 2-year period in a sample of 33 preschool-aged boys with FXS [130]. The percentage of boys who obtained $T$ scores in the clinical range also increased from 9 to $12 \%$ over the same period. However, considering that the norms of the CBCL were not developed for children with ID, the reliability of this scale for individuals with FXS is unknown, and it is unclear whether this scale can be useful in clinical trials given its narrow range of scores.

(4) The SNAP-IV [40]. This is a revision of the Swanson, Nolan and Pelham (SNAP) Questionnaire [131]. The SNAP-IV is an 18-item questionnaire addressing symptoms of ADHD (72 additional items cover ADHD-related behaviors such as those of Oppositional Defiant Disorder). It has been used in a completed (ampakine CX516) study [40] and an ongoing (ganaxolone) trial in FXS, showing very good testretest reproducibility but no sensitivity to change in the CX516 trial [40].

Overall, all these potential instruments for evaluating ADHD symptoms in FXS are promising shorter-term outcome measures, but their tool quality fall in the limited to no evidence available quality range.

\section{Conclusions}

Available ADHD rating scales seem particularly suited to studies evaluating shorter-term effects in FXS (Table 3); their measurement properties are at the limited to moderate tool quality range (Table 3 ). This evaluation may change after ADHD measures are fully evaluated in intervention studies in FXS.

\section{Anxiety \\ Background}

Anxiety-like behaviors in FXS are prevalent and frequently severe [3, 132]. Two features distinguish anxiety in FXS; its prominent social component and close relationship with and difficult differentiation from hyperarousal. Until recently, most data on anxiety in FXS was based on informal observations. The ABC-C has been the only standardized measure employed in a systematic way to characterize social anxiety, usually in the context of a general assessment of problem behaviors. As for other disorders associated with ID, delineating and quantifying anxiety in FXS is challenging because of the limited behavioral repertoire of individuals with cognitive impairment and their inability to report emotional states. Interestingly, in FXS anxiety-like behaviors seem to be stable across the age spectrum [103]. This suggests that, as in the general population, anxiety-like behaviors tend to be less dynamic than other problem behaviors and, consequently, instruments measuring them may be able to detect either shorter- or longer-term changes. Most standardized data on social anxiety in FXS has been collected using the $\mathrm{ABC}-\mathrm{C}$, which in its original form includes a subscale covering a variety of abnormal social interaction behaviors (i.e., ABC-C Lethargy/Social Withdrawal) [104]. Among this subscale's items, there are some corresponding to social anxiety. These were initially characterized as such by observation $[19,28]$ and, more recently, by factor analysis that generated the new Social Avoidance subscale of the FXS-specific $A B C-C_{F X}[111]$. Although the overall psychometric properties of the $\mathrm{ABC}-\mathrm{C}_{\mathrm{FX}}$ are good to excellent [103, 111], the fact that the Social Avoidance subscale includes only four items may limit its sensitivity (see Berry-Kravis et al., in press) [113]. This concern is also extended to the $A B C-C_{F X}$ Inappropriate Speech subscale. Nonetheless, the ABC- $C_{F X}$ Social Avoidance did show sensitivity to treatment in a randomized placebocontrolled phase 2 arbaclofen trial [112]. Since items directed at other forms of anxiety are not included in the $\mathrm{ABC}-\mathrm{C}_{\mathrm{FX}}$, different measures have been explored and considered potentially suitable but their sensitivity is still 
unknown. The Anxiety, Depression and Mood Scale (ADAMS) [133] was developed as a tool for screening anxiety and mood symptoms in individuals with ID (Table 3). The ADAMS has shown convergent validity with a caregiver diagnostic interview for anxiety in FXS [132] and psychometric evaluations in adults with ID revealed adequate internal consistency and convergent and discriminant validity $[115,133]$. Nonetheless, ADAMS's sensitivity to interventions is unknown. The Working Group also identified the Pediatric Anxiety Rating Scale (PARS) [134] as a promising measure, based on its successful application to selective serotonin reuptake inhibitor trials in pediatric anxiety disorders (Table 3). Other measures employed for measuring anxiety-like behaviors in FXS include the CBCL's Withdrawn subscale [19, 135] and CBCL's DSM-Anxiety subscale [136, 137]. Psychometric properties of these CBCL subscales in FXS, including their sensitivity to change, are unknown. Nonetheless, because of its content, more adequate for individuals in the normal or borderline IQ range, and limited range of scores, the CBCL's use in FXS clinical trials may be limited.

\section{Progress and plans in FXS}

(1) Pediatric Anxiety Rating Scale (PARS). A pilot observational study of the PARS in FXS, involving 49 subjects, aged 5-35 years, confirmed its potential for clinical trials. It demonstrated good psychometric properties, including feasibility, reliability, and convergent validity [138]. Responsiveness to change (i.e., sensitivity) is still unknown, although this information may become available soon since the PARS has been already implemented in three clinical trials.

(2)The Anxiety, Depression and Mood Scale (ADAMS). A recent observational study of the ADAMS in Rett syndrome concluded that, among measures of anxiety-like behaviors, it has the best psychometric properties [17]. Although sensitivity was not examined in this investigation, it included an assessment of the ADAMS' functional relevance. ADAMS scores correlated inversely with adaptive behavior skills and quality of life scores [17] indicating that this is a clinically meaningful measure for interventions (as defined by the FDA) for Rett syndrome and probably other neurodevelopmental disorders such as FXS. Moreover, a pilot open-label trial with mecasermin (recombinant human IGF-1) in Rett syndrome demonstrated that the ADAMS Social Avoidance subscale's was mildly sensitive to response to treatment [139]. A recently completed but not yet reported trial of ganaxolone in FXS employed, in addition to the PARS, the ADAMS.

\section{Potential measures}

As mentioned in the "Problem behaviors: focus on disruptive domain" section, review of the ID and ASD literature supports the need for anxiety measures with better psychometric properties, including greater sensitivity to interventions. The comprehensive examination of tools to measure outcomes in young children with ASD, conducted by the UK's NHS National Institute for Health Research, also covered anxiety [10]. The review found literature on eight measures for high-functioning children with ASD. Of these questionnaires, only three had strong measurement properties: the Spence Children's Anxiety Scale (SCAS), the Revised Children's Anxiety and Depression Scale (RCADS; revised SCAS), and the Screen for Child Anxiety Related Emotional Disorders (SCARED). Despite this, no data are available on the sensitivity of these three measures to change or intervention [10]. A similar review of anxiety measures for clinical trials involving individuals with ASD, an effort sponsored by Autism Speaks, concluded that, of 10 reviewed instruments, only four were considered clinically relevant and adequate "with condition" [13]. This meant that, despite adequate reliability and validity, not all psychometric properties were present (e.g., limited information on test-retest reliability). The four measures were the Child and Adolescent Symptom Inventory-4th Edition Revised (CASI-4R), the Multidimensional Anxiety Scale for Children (MASC), the Anxiety Diagnostic Interview Scale for DSM-IV (ADIS), and the aforementioned PARS [13]. Two measures evaluated positively in the British review, namely the RCADS and the SCARED, as well as the ADAMS were determined by Lecavalier and colleagues to have potential for clinical trials [13]. Both reviews recommended the development of outcome measures with better properties for trials focused on anxiety in ASD. Although some of the measures identified in these reviews on ASD are adequate for individuals with FXS, the content of others is not compatible with a level of functioning on the moderate to severe range of ID.

\section{Conclusions}

Evidence for the PARS and ADAMS reviewed here support their promising status as moderate quality tools, in part because of the lack of information on sensitivity to change. As indicated above, these instruments could be used for measuring either shorter- or longer-term outcomes. Until these tools are fully evaluated and shown to detect change in intervention studies, it will not be clear if they are adequate as primary endpoints for the assessment of anxiety in FXS. A few other measures, such as the RCADS and the SCARED, could be potentially useful in higher functioning individuals with FXS (i.e., most females). Nevertheless, considering that 
anxiety is a major behavioral abnormality in FXS, development of anxiety measures with better psychometric properties is still a worthwhile goal.

\section{Autistic behavior Background}

In DSM-5, ASD is characterized by qualitative impairments in social interaction and communication, as well as restricted interests and repetitive behaviors (RRBs; [39]). RRBs are also prevalent in ID without ASD. Therefore, RRBs that are mainly linked to cognitive impairment are addressed in the following subsection. The inclusion of a number of explicit specifiers, among them cognitive and language abilities, is a major change to the definition of ASD under the DSM-5 criteria that affects both individuals with idiopathic ASD and those with FXS who show severe autistic behaviors. Establishing the diagnosis of ASD can be a challenge in FXS, as in ID in general, since demonstrating the selectivity of the social interaction impairments (i.e., beyond overall cognitive or language impairment) can be very difficult $[2,16]$. This issue has been recognized in an expert consensus document on clinical practice from the Fragile X Clinical and Research Consortium [140] and a publication on ASD in FXS (see Kaufmann et al., in press) [141]. As in the general population with ASD [142, 143], severity of autistic behavior in FXS is variable and associated with age and IQ $[18,144]$. Severity of autistic behavior tends to be stable [103] or improve [144, 145] over time. However, one study reported increase in autistic behavior with age, as measured with the Childhood Autism Rating Scale (CARS; [146]) that contrasted with most other studies using gold standard instruments (e.g., Autism Diagnostic Interview-Revised (ADI-R); [144]). Therefore, the specific instrument used in the assessment of autistic behavior may influence the outcome of studies in FXS.

\section{Progress and plans in FXS}

For the reasons stated above, the Working Group endorsed efforts at establishing the reliability, validity, and sensitivity to change of several available measures of autistic behavior in FXS. Most of this proposed work has not yet been carried out. Therefore, the following section reviews the potential of several relatively well-established measures of autistic behavior for evaluation of individuals with FXS. We also briefly discuss efforts at determining outcomes in idiopathic ASD that may also lead to improvements in autistic behavior assessment in FXS.

\section{Potential measures}

McConachie and colleagues provided the most comprehensive to date review aimed at identifying the quality of tools used for measuring outcomes for young children with ASD, a project of the UK's NHS National Institute for Health Research [11]. A total of 17 measures of autistic behavior severity, 7 measures of social awareness, 4 tools for evaluating RRBs, and 2 instruments for specifically evaluating outcome of interventions, as well as instruments for other cognitive and behavioral domains, were included in this report. A parallel effort sponsored by the Autism Speaks foundation examined measures in terms of their adequacy for clinical trials in idiopathic ASD. Two of the publications resulting from the latter endeavor are pertinent to this section. The first evaluated 38 measures of social communication, concluding that only 6 were appropriate for use as outcomes [14]. The second focused on RRB; 24 measures were examined and 5 were recommended for use "with conditions" [15]. Although some of the instruments were included in more than one review, the number of assessed measures and the depth of their analyses preclude their discussion here. Nonetheless, these reviews are certainly the most important systematic work of relevance to autistic behavior in FXS published since the 2013 Report. For details, we refer the reader to the original publications.

Overall, the authors concluded that only sparse evidence exists on reliability and validity for only a few of the tools used in young children with ASD, probably the group of greater relevance to FXS. Since our literature search revealed that only in idiopathic ASD there has been a systematic review of the tools in terms of measurement properties, we focus on those instruments without having separate sections for ASD in FXS and idiopathic ASD.

Table 4 lists measures of autistic behavior severity and RRB other than autistic behaviors, including their suitability for shorter-vs. longer-term clinical trials and quality of measurement properties using the COSMIN grading criteria. We review these instruments here and in the next section.

(1)Autism Diagnostic Observation Schedule-Generic (ADOS-G). The ADOS-G is a widely used and wellestablished semi-structured, interactive instrument designed to assess aspects of social reciprocal interaction, communication, stereotyped behaviors, and restricted interests and play [147]. It is included in this review because the ADOS-G Severity Score is an overall measure of autistic behavior severity [148] and it has been administered pre- and post-treatment in the Early Start Denver Model (i.e., early intervention) randomized controlled trials in idiopathic ASD [149, 150]. There have been some recent refinements of the ADOS-G algorithm, such as the development of the ADOS-Calibrated Severity Score (ADOS-CSS) [148] that is independent of age, IQ, and language level [148, 151, 152]. The ADOS-G and, in particular, the ADOS-CSS seem to be suited for shorter-term trials 
$[149,150]$. Together, based on data on reliability [147, $153-155]$ and validity $[147,154-158]$, the tool quality of the ADOS measures is in the limited to moderate range in idiopathic ASD. However, because of the lack of studies in individuals with the disorder, ADOS measures should be considered as having limited tool quality for FXS.

(2)Social Responsiveness Scale (SRS). The features of the SRS were reviewed in the preceding section on "Social cognition". A single open-label 10-week trial of acamprosate involving 12 subjects with FXS, aged 617 years, demonstrated a significant improvement in the scores of the SRS and several other cognitive and behavioral measures [159]. As noted in the "Social cognition" subsection under the "Cognitive measures" section, the SRS is suited for evaluating either shorterterm or longer-term effects, and its tool quality should be considered in the limited-moderate range in FXS.

(3) Childhood Autism Rating Scale (CARS). This is a widely used 15-item observation and parent/caregiver interview that quantifies the severity of behaviors associated with ASD, with total scores $\geq 30$ strongly suggesting the presence of the disorder [160, 161]. The CARS, Second edition (CARS-2) is a more recently published clinician-completed rating also used to determine ASD symptom severity [162]. The CARS-2 is suitable for evaluating shorter-term effects; its reliability [160, 163, 164], validity $[165,166]$, and lack of application to trials in FXS place it at the limited tool quality level.

Table 4 Outcome measures of autistic and non-autistic repetitive behaviors in fragile $X$ syndrome

\begin{tabular}{clll}
\hline Domain $\begin{array}{l}\text { Outcome } \\
\text { measure }\end{array}$ & $\begin{array}{l}\text { Shorter } \\
\text { term }\end{array}$ & $\begin{array}{l}\text { Longer } \\
\text { term }\end{array}$ & Quality of tools \\
\hline Autistic behavior & & & \\
ADOS & Yes Yes & $+[147-155]$ \\
SRS & Yes & $+/++[15,46,78-81,112,113]$ \\
CARS & Yes & $+[160-166]$ \\
SCQ & Yes & $+[162,167,173,174]$ \\
RBS-R & Yes & $+[15,179-184]$ \\
Repetitive non-autistic behavior & \\
RBS-R & Yes & $+[15,66,179,180,184,191,192$, \\
& & $194]$ \\
RBQ & Yes & $+[189]$ \\
BPI-S & Yes & $+[179,195,196]$
\end{tabular}

Abbreviations: ADI-R Autism Diagnostic Interview-Revised, ADOS Autism Diagnostic Schedule, SRS Social Responsiveness Scale, CARS Children Autism Rating Scale, SCQ Social Communication Questionnaire, RBS-R Repetitive Behavior Scale Revised, RBQ Repetitive Behavior Questionnaire, BPI-S Behavior Problems Inventory, Short Form, Shorter term deemed suitable for clinical trials lasting $<12$ months, Longer-term deemed suitable for clinical trials lasting $>=12$ months, Grading system: +++ strong evidence, ++ moderate evidence, + limited evidence, ? unknown due to poor methodological quality, blank cell no evidence available
(4) Social Communication Questionnaire (SCQ; originally called the Autism Screening Questionnaire; [167]). The SCQ is a 40-item parents/caregivers rating scale based on the ADI-R [168] that screens for current core ASD behaviors and at age 4-5 years (lifetime). Language items not suitable for non-verbal children can be omitted; it is scored according to language level (maximum score 32 or 39), with higher scores indicating more severe symptoms. Five studies have used the SCQ to characterize autistic behavior and its severity in FXS [141, 169-172]. However, none has examined measurement properties including sensitivity. The SCQ is adequate for evaluating shorterterm outcomes. SCQ's reliability [173, 174] and validity ([163]; reviewed in [12]) support the limited tool quality level.

(5) Repetitive Behavior Scale-Revised (RBS-R). RRBs are a broad range of behaviors that are subdivided into two conceptual categories [175]: "lower-order" motor actions associated with lower developmental levels and characterized by repetition of movement (e.g., dyskinesias, stereotyped and repetitive manipulation of objects, and repetitive forms of self-injurious behavior (SIB)), and more complex or "higher-order" behaviors associated with higher cognitive abilities (e.g., object attachments, insistence on sameness, repetitive language, and circumscribed interests) [176, 177]. Both categories of behavior appear to be a function of an overall cognitive/behavioral rigidity/lack of flexibility [178]. Although no single RRB appears to be specific to ASD, an elevated pattern of RRB occurrence, cooccurrence, and severity characterizes the disorder [179]. The RBS- $\mathrm{R}$ is the most widely applied instrument for evaluating RRBs. The RBS- $\mathrm{R}$ is a 43-item parent/caregiver rating scale that was normed on individuals with ID and revised to capture some of the complex RRBs observed in ASD [179]. The items have been grouped into six subscales: Stereotyped, Selfinjurious, Compulsive, Ritualistic, Sameness, and Restricted interests. The RBS-R was designed for use in populations with ASD or related neurodevelopmental disorders $[179,180]$. RBS-R's factor structure has been empirically evaluated in multiple studies [181-184]; recently, Bishop and colleagues concluded that a fivefactor structure (Sensory Motor, Restricted Interests, Self-injury, Compulsive, and Ritualistic/Sameness and Sensory Motor behaviors) was the best solution for the RBS-R [183]. The scale's psychometric properties are variable, ranging from poor (test-retest reliability) to strong (internal consistency) [180]. Thus, its overall quality as tool in idiopathic ASD should be considered moderate. The RBS-R was applied in a study that found different profiles of RRBs in young boys, aged 3-5 years, with either FXS and ASD or idiopathic 
ASD [185]. Both groups were similar with respect to lower-order (motoric) RRBs, but differed in terms of more complex forms that were less severe in FXS with ASD [186]. Furthermore, Wolff and colleagues found positive correlations between Self-injurious total scores on the RBS-R and number of SIB topographies and bilateral caudate nuclei volumes in the FXS group comorbid for ASD [187]. This study also reported that the profile of RRBs remains stable in FXS from preschool through at least the middle school years. This is in contradiction with the analyses of the $\mathrm{ABC}-\mathrm{C}_{\mathrm{FX}}$ Stereotypy, which demonstrates a decline in scores over time [103]. To date, the only FXS-specific factor analyses conducted on any measure of RRBs are those performed for the development of the $\mathrm{ABC}-\mathrm{C}_{\mathrm{FX}}$. Although the RBS-R has been used in some FXS trials, information on its sensitivity to change is not yet available. Thus, the RBS-R seems suited for evaluating shorter-term and possibly also long-term effects. In the review of measures of RRBs for trials in idiopathic ASD by Scahill and colleagues, the authors concluded that the RBS-R, as well as the ABC-C Stereotypy subscale, Stereotyped Behavior Scale (SBS), and the Repetitive Behavior Questionnaire (RBQ) (see the next section), are appropriate with conditions [15]. Based on the latter and the lack of data in FXS, the RBS-R's tool quality should be considered limited. However, because of the relevance of RRBs to the FXS behavioral phenotype, the RBS-R and similar instruments should be further studied in terms of suitability for clinical trials for this genetic disorder.

\section{Conclusions}

The measures of autistic behavior reviewed here seem adequate as shorter-term endpoints. Although they show moderate-strong measurement properties in individuals with idiopathic ASD, the evidence is limited in FXS and their sensitivity to change in this population is unknown to large extent because core autistic behaviors have not been the target of trials in FXS.

\section{Repetitive behaviors other than autistic behavior Background}

Numerous behaviors included in the RRB category are frequently observed in a wide range of neurodevelopmental and neuropsychiatric conditions, not only ASD [188-190]. In this section, we focus on instruments for RRBs pertinent to ID only, with emphasis on the FXS phenotype. Available information on the differences in RRBs between ID and idiopathic ASD suggest that they are mainly quantitative [179]. Limited evidence suggests that FXS is associated with increased risk for RRBs. Indeed, perseverative speech is a hallmark feature often problematic in boys with the disorder [66, 191, 192].
Other RRBs that are also elevated in FXS in general include hand flapping, body rocking, and SIB [193]. Different measures have been used to assess the severity of RRBs in FXS. They include specific instruments such as the RBS-R [179] and components of tests developed for measuring overall problem behaviors $\left(\mathrm{ABC}-\mathrm{C}_{\mathrm{FX}} ;[111]\right)$. The Working Group endorsed efforts at establishing the reliability, validity, and sensitivity of several currently available measures of RRBs other than autistic within the FXS population.

\section{Progress and plans in FXS}

(1) Repetitive Behavior Scale-Revised (RBS-R). Although RRB categories have been reported in FXS at a global level, there is limited research characterizing the relative frequency of different types of RRBs or association with other factors. The RBS-R, discussed in more detail in the previous section on "Autistic behavior," was employed by Oakes and colleagues to examine the profile of RRBs in 39 boys with FXS, aged 6-10 years, without specifying their ASD status [194]. Restricted Interests and Sensory Motor behaviors were reported as most problematic, in contrast to SIB. Non-verbal IQ was negatively related to RRBs in general, whereas anxiety and social affective symptoms of ASD were positively correlated with scores on Restricted Interests. Anxiety was also positively correlated with scores of Compulsive behaviors and Ritualistic Sameness behaviors. Despite the relative specificity of the reported profile [194], it is important to point out that all subscales of the RBS-R were significantly inter-correlated. No application of the RBS-R for measuring changes in non-ASD-related RRBs has been reported. Therefore, this particular use of the RBS-R cannot be evaluated or a tool quality grading of no evidence available is the most appropriate.

(2)ABC-C Stereotypy and Inappropriate Speech subscales. These are two of the five original subscales of the ABC-C that cover RRBs. The content of both subscales was relatively preserved after the factor analysis that originated the $\mathrm{ABC}-\mathrm{C}_{\mathrm{FX}}$ (i.e., one Stereotypy item was incorporated into the new Socially Unresponsive/Lethargic subscale; [111]), demonstrating their appropriateness for FXS. Two independent studies demonstrated the usefulness of these subscales for delineating trajectories of RRBs in FXS [103, 111]. While these cross-sectional analyses showed that behaviors under Stereotypy are in the moderate range of severity and tend to slightly decrease over time, those under Inappropriate Speech are on the severe range and remain relatively stable between 6 and 25 years. Since the $A B C-C_{F X}$ autistic behavior-oriented Socially Unresponsive/Lethargic subscale did not incorporate 
many items of Stereotypy and Inappropriate Speech, it is assumed that these subscales reflect predominantly non-autistic RRBs. However, no direct evidence supporting this is currently available. As the $A B C-C_{F X}$ Inappropriate Speech subscale includes only four items; this may limit its sensitivity in a similar fashion to the Social Avoidance subscale (see the "Anxiety" subsection, under the "Behavior and Emotion measures" section). We can conclude that, as other $\mathrm{ABC}-\mathrm{C}_{\mathrm{FX}}$ subscales, Stereotypy and Inappropriate Speech are adequate for assessing either shorter-term or longer-term outcomes. Psychometric data, including their application to several recent FXS trials, support a moderate general tool quality label. Nonetheless, no data are available on the specific use of these instruments for measuring non-autistic RRBs in FXS. Therefore, their tool quality for this specific indication would correspond to no evidence available.

\section{Potential measures}

(1) Repetitive Behavior Questionnaire (RBQ). The RBQ is a 19-item scale that shares some individual items with the RBS-R. Moss and colleagues examined the presence of RRBs across six groups of individuals with ID, including males with FXS $(n=191)$, aged 6-47 years. It was reported that the FXS group had higher scores on all five subscales (Stereotyped Behavior, Compulsive Behavior, Insistence on Sameness, Restricted Preferences, and Repetitive Speech); moreover, they also had more severe RRBs than two other ID groups in the categories of Compulsive Behavior, Insistence on Sameness, and Repetitive Speech [189].

(2) Behavior Problems Inventory (BPI). Employing an earlier version of the BPI scale [195], an instrument described in the "Problem behaviors: focus on disruptive behavior domain" subsection, and the RBS, Bodfish and colleagues found that the difference in RRBs between ID and ASD was mainly quantitative [179]. Namely, they reported that the occurrence of specific topographies of RRBs as well as their severity in 34 adults (23 males, 11 females) with non-ASD ID (CARS <30) matched to that of an ASD group. This finding supports the notion that the RRBs in ASD are similar to those described in ID [196]. While both groups, ID and ASD, had significant patterns of RRBs co-occurrence, the exception was the frequency of dyskinesias that was higher in the ASD group.

Although the data on the RBQ and the earlier version of the BPI support the notion that instruments measuring RRBs in ASD are adequate for FXS and other forms of ID, no data on the use of these instruments in clinical trials in FXS is available.

\section{Conclusions}

While the RBS-R and the ABC-CFX Stereotypy and Inappropriate Speech subscales present many adequate attributes and they have been already applied to intervention studies in FXS, their adequacy for measuring RRBs other than autistic behaviors has not been formally assessed. Therefore, further testing other suitable measures, such as the RBQ and BPI, or developing new ones could still be a worthwhile effort.

\section{Overall conclusions on Behavior and Emotion measures}

While the main targets of intervention studies in FXS have been maladaptive/problem behaviors, and these overlap with ADHD-like behaviors and RRBs, anxiety and autistic behaviors are also a major concern. Over the years, this situation has raised the question whether it is possible to develop a comprehensive instrument covering all abnormal behaviors in FXS. The ongoing application and refinement of the FXSRS [114], during the course of trofinetide trials in FXS, represents the most recent effort in this area. This instrument is a traditional clinician rating scale. While it is too early to determine the value of the FXSRS, the utility of a single "everything" measure is obvious. Nonetheless, the pervasive use of devices such as smart phones, tablets, and wearable sensors in behavioral and mental health research [197, 198] begs the question of when would be appropriate to incorporate technologies to behavioral assessments in FXS, including in the context of clinical trials. These novel technologies may allow more naturalistic, situation-related, and multiple sampling of behavioral abnormalities, perhaps with greater implications for quality of life, in FXS and other neurodevelopmental disorders. Thus, paradigms for adequate validation of such novel measures would be an important contribution to the field.

Finally, the issue of biologic validation of behavioral measures, recommended in the 2013 Report through correlations with valid FXS biomarkers, remains pending due to the slow progress in the Biomarkers/Medical domain as reviewed in the following domain and in the "Discussion" section.

\section{Biomarkers and Medical measures}

The Working Group endorsed further effort toward validating existing biomarkers as critically needed measures for development in the field. Their objective nature and closer link to CNS function make them particularly attractive as outcome measures. The Biomarkers group includes a wide range of measures, from the relatively easy-to-apply blood-based biomarkers to those providing more direct evidence of CNS function such as neurophysiological and neuroimaging methods. In spite of 
their wide application, none had well-established clinical correlations or validity at the time of the 2013 Report.

Table 5 lists potential biomarkers classified in terms of their suitability for shorter- vs. longer-term outcomes and COSMIN quality grading system.

\section{Blood and tissue biomarkers Background}

Deficient FMRP expression has significant downstream consequences disrupting many cellular signaling pathways. As mentioned above, this is a particularly attractive group of measures because of their minimally invasive nature and easy application.

\section{Progress and plans in FXS}

Blood-based analyses hold promise as minimally invasive windows into cellular dysregulation secondary to deficient FMRP production occurring both in the periphery and, at least some, regions of the brain. Several molecular and biochemical blood assays have been evaluated in the context of published FXS-specific drug trials.

(1)Mitogen-activated protein kinases/extracellular signal-regulated kinases (MAPK/ERKs). The MAPK/ ERKs are a group of proteins that regulate nodal points for several signaling cascades. MAPK/ERK regulation mediated by phosphorylation is typically termed activation. Delayed early-phase phosphorylation of ERK has been noted in lymphocytes of individuals with FXS and in neurons and thymocytes of Fmr1 knockout mice. This delay is described as an enhanced time to half maximum ERK activation following phorbol ester stimulation [199]. ERK peripheral lymphocytic activation has been assessed pre- and post-drug treatment in studies of lithium and riluzole in FXS. ERK activation was significantly reduced (i.e., normalized) following lithium [55] or riluzole [200] administration. In an open-label trial involving 16 individuals with FXS, aged 6-23 years, lithium (a mood stabilizer that inhibits the phospholipase C signaling pathway and GSK-3B [201]) use was associated with normalization of ERK activation kinetics but no improvement in maladaptive behaviors evaluated by the ABC-C [54]. These findings can be explained, at least in part, because of the high variability of the biochemical assay (Berry-Kravis, personal communication). Riluzole is FDA approved for treatment of amyotrophic lateral sclerosis and is postulated to reduce glutamate release, block NMDA receptors, and enhance GABA activation [202]. In this pilot 6-week trial including six males with FXS, aged 19-24 years, Erickson and colleagues found that despite normalization of ERK activation kinetics, no significant clinical improvements were noted [200]. A recent evaluation of ERK and Akt (another major kinase pathway) phosphorylation in individuals with FXS, in relation to lovastatin treatment, demonstrated normalization of baseline increased ERK activity after drug administration. Of note, these changes in ERK phosphorylation were correlated with clinical response to lovastatin [203].

(2)Brain-derived neurotrophic factor (BDNF). BDNF is a growth factor and synaptic modulator that supports survival, growth, and differentiation of neurons. BDNF has been shown to impact FMRP expression [204]; its application to hippocampal slices from Fmr1 knockout mice leads to rescue of long-term potentiation deficits [205]. BDNF expression has also been noted to be reduced in Fmr1 knockout mice [206]. In a study of acamprosate in 12 individuals with FXS, aged 5-17 years, BDNF plasma levels were shown to increase significantly after 10 weeks of treatment with the drug [159]. Nonetheless, BDNF levels in the nine subjects deemed to be responders did not correlate with their clinical response. Acamprosate is drug approved for the treatment of alcoholism, which is postulated to attenuate glutamatergic activity, particularly NMDA receptor-dependent, and potentiate GABA A receptor activity [207]. Additional relevant data on BDNF were obtained in a small open-label fluoxetine trial in children and adolescents with idiopathic ASD. Using the Autism Treatment Evaluation Checklist, the cohort showed improvements in several aspects of communication, socialization, and cognitive awareness, changes that were correlated to decreases in serum levels of BDNF [208]. Since the direction of the BDNF changes was unexpected, this study emphasizes the need for further exploration of BDNF and related molecules as biomarkers of response to treatment in neurodevelopmental disorders including FXS.

(3) Amyloid precursor protein (APP). APP is a key neural modulator, which is processed by two pathways. The best known is the so-called amyloidogenic or $\beta$ pathway that leads to the production of the neurotoxic $A \beta 40$ and $A \beta 42$ fragments, which have been implicated in the pathophysiology of Alzheimer disease. The non-amyloidogenic or $\alpha$ pathway results in the production of the soluble neurotrophic APP alpha (sAPPa) [209]. FMRP is known to regulate APP mRNA expression [210, 211], with baseline APP levels being elevated in Fmr1 knockout mice [211]. APP and its cleavage products were measured in a subset of nine participants (mean age 10.9 years) in the acamprosate trial described above [159]. Total APP and sAPP $\alpha$ were reduced after treatment, while $A \beta 40$ and A $\beta 42$ remained unchanged. These APP-related decreases correlated modestly with improvements on the ABC-C Lethargy/Social Withdrawal subscale. 
Table 5 Potential biomarker measures in fragile $X$ syndrome

\begin{tabular}{|c|c|c|c|c|}
\hline Domains & Potential outcome Measure & Shorter term & Longer term & Quality of tools \\
\hline \multicolumn{5}{|l|}{ Blood and tissue } \\
\hline \multirow[t]{2}{*}{ FMR1/FMRP expression ${ }^{a}$} & FMR1 methylation & Yes & Yes & $+[9,113,261]$ \\
\hline & FMRP levels & Yes & Yes & $+[9,113]$ \\
\hline \multicolumn{5}{|l|}{ Signaling pathway } \\
\hline & ERK activation rate & Yes & Yes & $+[54,55,200,203]$ \\
\hline & BDNF level & Yes & Yes & $+[159,204,208]$ \\
\hline & APP and metabolites levels & Yes & Yes & $+[159,209]$ \\
\hline & MMP activity & Yes & Yes & $+[87,227]$ \\
\hline & mTOR activity & Yes & Yes & $+[269]$ \\
\hline & S6 Kinase activity & Yes & Yes & $+[269]$ \\
\hline \multicolumn{5}{|l|}{ Neurophysiological } \\
\hline & Electroencephalogram & Yes & & $+[217,218,227,228,232,233]$ \\
\hline & Event-related potentials & Yes & Yes & $+/++[219,222-224,227-230]$ \\
\hline & Prepulse inhibition (PPI) & Yes & & $+/++[6,117,226]$ \\
\hline & Eye tracking and pupillometry & Yes & & $++[77,85-88,139,216]$ \\
\hline \multirow[t]{10}{*}{ Neuroimaging } & MRI & & & \\
\hline & sMRI & Yes & Yes & $+[235]$ \\
\hline & $-\mathrm{vMRI}$ & Yes & Yes & $+[237-240]$ \\
\hline & $-D W l$ & Yes & Yes & $+[241-243]$ \\
\hline & Functional MRI & & & \\
\hline & $-f M R I$ & Yes & Yes & $+[244-250]$ \\
\hline & -rs-fMRI & Yes & Yes & $+[77]$ \\
\hline & -MRS & Yes & Yes & $+[270]$ \\
\hline & -pMRI & Yes & Yes & $+[234]$ \\
\hline & NIRS & Yes & & $+[251-255]$ \\
\hline
\end{tabular}

Abbreviations: FMR1 fragile X mental retardation 1 gene, FMRP fragile $\mathrm{X}$ mental retardation protein, ERK extracellular signal-related kinases, $B D N F$ brain-derived neurotrophic factor, APP amyloid precursor protein, MMP matrix metalloproteinases, mTOR mechanistic target of rapamycin, MRI magnetic resonance imaging, pMRI perfusion MRI, $s M R I$ structural MRI, $v M R I$ volumetric MRI, DWI diffusion-weighted imaging, $f M R I$ functional $\mathrm{MRI}, r s-f M R I$ resting state $f M R I, M R S$ magnetic resonance spectroscopy, NIRS near-infrared spectroscopy, Shorter term deemed suitable for clinical trials lasting $<12$ months, Longer term deemed suitable for clinical trials lasting >=12 months. Grading system: +++ strong evidence, ++ moderate evidence, + limited evidence, ? unknown due to poor methodological quality, blank cell no evidence available

${ }^{a}$ Evaluated as a predictor of response

\section{Potential measures}

A variety of blood-based biomarkers are being considered in translational research in the idiopathic ASD field. They include among others markers of immune processes [212], inflammation [213], oxidative metabolism [214], and the serotoninergic system [215]. It is possible that, as BDNF and APP, these ASD-related biomarkers will become eventually applicable to FXS trials.

\section{Conclusions}

The body of literature on blood biomarkers in FXS continues to increase; however, changes are still inconsistent or unrelated to clinical effects. Only the ERK activation assay has been replicated, and most studies have involved small cohorts. Not only larger-scale replication investigations but also studies exploring other biomarkers are needed, since the impact of deficient FMRP are wide-reaching and likely affecting many cellular pathways that could be potentially evaluated in peripheral blood. Thus, in terms of tool quality, we consider bloodbased biomarkers limited (BDNF, amyloid markers, ERK). Because of their dynamics, these biomarkers are adequate for evaluating shorter-term effects in FXS. Nevertheless, follow-up studies may determine their appropriateness as longer-term measures.

\section{Neurophysiological measures Background}

There is a critical need for objective direct measures of CNS function that can provide feedback about a drug's engagement to its target. Although potentially valuable as non-invasive measures of treatment response, blood 
biomarkers may not directly reflect CNS function, as receptors and other molecules targeted by drugs are frequently either not expressed in blood cells or linked to different signaling cascades than in the CNS. The Working Group recommended (1) to correlate biomarkers that measure brain activity directly such as prepulse inhibition of the startle response (PPI), eye tracking/pupillometry, and functional MRI (fMRI) with measures of neurobehavioral function in individuals with FXS in order to establish clinical validity [6]. The need for new markers of CNS engagement has been made even more apparent by the recent challenges in seeing significant changes in behavior with drugs targeting synaptic dysfunction in FXS ("failed trials").

In the 2013 Report, it was noted that PPI was the most extensively studied electrophysiological measure of relevance to clinical trials in FXS. PPI reflects sensory inhibitory gating, which is regulated by mGlur5 signaling and deficient in FXS [117]. In addition, PPI has good test-retest reliability and a similar profile in individuals with FXS and Fmr1 knockout mice [117]. All these features make PPI a promising biomarker in FXS.

Eye gaze abnormalities are well documented in FXS; gaze avoidance is a hallmark behavioral feature of the disorder, with affected individuals having difficulty in establishing and maintaining eye gaze during social interactions [2]. Pupillary responses, regulated by the autonomic nervous system in part in response to level of emotional arousal, are also abnormal in FXS [85, 216]. Eye-tracking devices such as the Tobii system have been successfully used to measure eye gaze aversion and pupillary responses in FXS $[85,216]$, supporting the notion that these could become biomarker-type of outcome measures in clinical trials.

Electroencephalogram (EEG) is one of the oldest noninvasive methods for evaluating brain function, by measuring on the scalp fluctuations in electrical activity that reflect neural network properties [217]. Despite its poor spatial resolution, EEG continues to be a valuable tool for research where millisecond-range temporal resolution is desired [218]. In the clinical context, one type of EEG parameter typically estimated with potential translational research use is spectral content (e.g., spectral power of different frequency bands) [210]. Other commonly used derivatives of EEG signal used in clinical and cognitive research, with translational potential, include evoked potentials (EPs) and event-related potentials (ERPs). EPs are estimated by averaging over EEG activity that is time-locked to the presentation of a stimulus (e.g., auditory tone or visual checkerboard). Similarly, ERPs are estimated by averaging over EEG activity that is time-locked to more complex higher-order cognitive processing of the stimuli (e.g., ERP related to response inhibition in a Go/No-Go experimental paradigm). In FXS, auditory ERP is known to be abnormal with a larger magnitude of response [219] in correspondence with the characteristic behavioral phenomenon of sensory over-reactivity (hyperarousal) to sound [220]. Over-reactivity to a variety of sensory stimuli is common in FXS and thought to represent circuit hyperactivation associated with increased dendritic spine density in sensory cortices [2]. A barrel cortex neuronal model for this phenotype has been described in the Fmrl knockout mouse [221]. As individuals with FXS, Fmr1 knockout mice also show a larger auditory response amplitude [222]. Thus, auditory ERP may not only be a direct window into synaptic dysregulation in FXS but also a highly attractive measure for direct translation of drug effects from mouse to man. ERP measures may also be relevant to the distinctive mGluR5 dysregulation in FXS, since the mGluR5 antagonist MPEP reduced N1 auditory EP amplitudes in a mouse model of ASD [223]. There is also evidence that ERP resting-state measures are modulated by inhibition of mGluR5 signaling by the mGlur5 antagonist mavoglurant [224]. Another ERP parameter, mismatch negativity (MMN) may also be a useful measure in FXS trials, as it has been reported to be an index of language impairment in ASD [225]. Additional information about the application of EGG-related to measures to FXS and background on PPI and eye tracking/pupillometry is included in the following section.

\section{Progress and plans in FXS}

(1)Prepulse inhibition (PPI). The potential of PPI as a biomarker in FXS was confirmed in an early trial $(n=12)$ with the mGluR5 antagonist fenobam that showed $a \geq 20 \%$ improvement in half of the subjects (although there was no clear clinical improvement) [226]. However, subsequent work with PPI reveals that the measure is very sensitive to differences in equipment and environment, which has led to lack of inter-site reproducibility. Consequently, PPI may be most useful in early proof-of-concept trials as opposed to large confirmatory multi-site trials. PPI seems suited for evaluating shorter-term effects, and its tool quality is limited-moderate for the aforementioned reasons.

(2)Eye tracking and pupillometry. A standardized protocol quantifying pupillary diameter and looking time and number of fixations to the eye region for a series of happy, calm, and fearful human faces has been shown to be suitable for individuals of varying ages with FXS, with a 7-51 years age range, with strong feasibility and test-retest reliability for all outputs [85]. This protocol has been applied in several clinical trials, although a full analysis of sensitivity to treatment has not yet been published. However, 
preliminary reports indicate normalization of parameters in response to treatment with minocycline [87] or the mGluR5 antagonist mavoglurant [88]. The eyetracking protocol described above is the only one employed in clinical trials in FXS; nonetheless, several others have been developed and are potentially useful (see the "Social cognition" section). In conclusion, although reports on ongoing studies may change our conclusions, at present, eye tracking and pupillometry should be considered moderate level quality tools suited for evaluating shorter-term effects in FXS trials.

(3) Event-related potentials (ERPs). Several observational and treatment studies, mainly pilot type, have utilized EEG to characterize ERPs in FXS and to assess treatment response and associated changes in cortical activity. Van der Molen et al. showed that the N1 and P2 components of auditory ERPs from 16 males with FXS, aged 18-42 years, were significantly enhanced. Schneider and colleagues observed changes in electrocortical habituation to auditory stimuli (changes between the first and last of 45 presented stimuli), specifically improved N1 and P2, associated with minocycline treatment (double-blind, placebocontrolled crossover, $n=12$, mean age 10.5 years), suggesting the feasibility and sensitivity of ERPs as a biomarker in FXS treatment trials [227]. In another study, Yang et al. employed auditory ERPs to evaluate the effects of chronic memantine treatment on verbal memory in individuals with fragile $\mathrm{X}$-associated tremor/ataxia syndrome (FXTAS) [228, 229]. The authors reported treatment-associated benefit on cuedrecall memory as well as corresponding changes in N400 ERP response [228, 229]. Recently, Ethridge and colleagues also examined ERPs during a somewhat different passive auditory habituation task in individuals with FXS ( $n=14,14-57$ years) and found "giant" N1 amplitudes to all four repeated stimuli in the FXS group more frequently than in a matched control subjects [230]. Of relevance to behavioral measures in FXS, the N1 amplitude enhancement was strongly correlated with auditory processing abnormalities on the Sensory Profile, a caregiver questionnaire employed in the ASD field that measures children's sensory processing abilities [231], and with increased ASD features on the SCQ [230]. N2 ERP amplitudes were also decreased in FXS and correlated with scores on the ABC-C Irritability subscale. Overall, this study [230] exemplifies the type of information provided by auditory ERPs in FXS that links biomarkers and behavioral outcome measures. No study so far has examined age- or gender-related differences in ERP profiles in FXS, and no data are available on testretest reliability or other measurement properties. However, studies are currently in progress to examine resting state and MMN in FXS. The use of ERP as a biomarker of multiple aspects of neural activity and processing in FXS is currently being explored at several centers. This includes the introduction of auditory ERP as a biomarker in the upcoming mavoglurant and language learning trial in children with FXS (U01 NS096767). Thus, at present, auditory ERP seems to be a biomarker suited to evaluating medication shorteror longer-term effects, with current evidence supporting a limited-moderate tool quality. Other potentially valuable neurophysiological measures, in particular EEG spectral analyses, are just beginning to be examined in FXS, as described in the following section.

\section{Potential measures}

EEG spectral analyses. These are being developed, and the recent pilot application of the frontal alpha band asymmetry index, a measure linked to anxiety and mood disorders, in a Rett syndrome trial testing full-length IGF-1 (mecasermin) suggests a good potential for EEG spectral analyses in FXS. IGF-1 treatment not only markedly reduced the right-sided abnormal asymmetry present in most subjects but these changes also correlated with reduction in anxiety severity [139]. Pilot studies have reported that gamma band amplitude and asymmetry could be biomarkers of response to behavioral interventions in $\operatorname{ASD}[232,233]$, and studies to evaluate this parameter are ongoing in FXS.

\section{Conclusions}

PPI is a biomarker with utility limited to early-phase trials. Both eye tracking and pupillometry are biomarkers of greater potential because they may be applicable throughout the continuum of clinical drug development. EEG-related measures such as auditory ERP and spectral analyses have great potential in FXS and are beginning to be tested in FXS trials. Other physiological measures already explored in neurodevelopmental disorders, such as actigraphy (accelerometer) studies of hand stereotypies in ASD and Rett syndrome, will be hopefully extended to FXS.

\section{Neuroimaging studies Background}

Magnetic resonance imaging (MRI) is a neuroimaging modality that uses magnetic field and radiofrequency waves for in vivo imaging of the CNS. Structural MRI techniques include volumetric MRI (vMRI) and diffusionweighted imaging (DWI) for mapping the morphology and white matter microstructure of the brain, respectively. Functional techniques include fMRI for measuring blood oxygenation level dependent (BOLD) signal, magnetic resonance spectroscopy (MRS) for measuring metabolic 
changes in the brain, and perfusion MRI (pMRI) for examining tissue perfusion [234]. A large number of MRI studies of individuals with FXS demonstrate that brain structure, function, connectivity, and metabolism are abnormal beginning early in development. For a review, see Fung and Reiss [235]. Some of these studies were conducted on a longitudinal basis, thereby providing initial "growth curves" of brain development in FXS, already available for typical development and other disorders [236]. Comparisons of brain growth curves from individuals with FXS with normative data could assist in the characterization of cohorts, including profiling responders, as well as providing information about treatment response in future trials.

\section{Progress and plans in FXS}

(1)Structural MRI. Research in other disorders indicates that significant changes in gray and white matter volume, white matter microstructure, and brain activation can occur in association with intervention within relatively short periods of time. For example, Roberto and colleagues showed that short-term (mean 50 days) weight restoration in under-weighed adult patients with anorexia nervosa was associated with significant increases in gray and white matter volume relative to healthy controls [237]. Eijk et al. demonstrated that, in individuals with alcohol abuse, 2 weeks of supervised abstinence was associated with significant recovery of gray matter volume in several brain regions including the cerebellum and parietal lobe [238]. There are many other examples of relatively rapid changes in neuroimaging metrics in clinical groups receiving disorderfocused intervention. Administration of risperidone and ziprasidone to individuals with schizophrenia led to increased cerebral gray matter volume after 28 days of treatment [239] and administration of citalopram to depressed patients increased hippocampal gray matter within 8 weeks [240]. Changes have also been observed in white matter microstructure by DWI; patients with multiple sclerosis showed increases in fractional anisotropy within 2 months of facilitation of physiotherapy [241], depressed patients showed increase in fractional anisotropy with 4 weeks of psychotherapy [242], and patients with obsessive-compulsive disorder showed a reversal of abnormal white matter microstructure after a 12-week course of antidepressant (SSRI) treatment [243].

(2) Functional MRI. The feasibility and utility of fMRI as a putative biomarker of intervention has been demonstrated in hundreds of treatment studies, as described in recent reviews of pharmacology, psychotherapy, pain management, and rehabilitation [244-250]. A recently completed study by Reiss and colleagues utilized fMRI as an outcome measure in a clinical trial of donepezil for FXS; these functional imaging data are currently being analyzed to determine if drug-related effects are observable and associated with clinical change.

\section{Potential measures}

Near-infrared spectroscopy (NIRS) is a method of diffuse optical brain imaging that uses light to non-invasively probe the cerebral cortex for changes in blood oxygenation related to brain function [251]. NIRS-based neuroimaging provides excellent temporal sensitivity, as well as reasonable spatial sensitivity. NIRS is relatively easy to set up, portable, and more tolerant to movement than MRI. Technological improvements and advancement in signal processing methods now enable scientists to employ NIRS to investigate a variety of hard-to-test clinical populations within naturalistic environments. The aforementioned advantages have resulted in the use of NIRS as a tool to monitor brain function related to clinical conditions including epilepsy, migraine, cerebrovascular disease, schizophrenia, ADHD, and others [252]. There is also emergent use of NIRS as an outcome measure in intervention studies in neurorehabilitation [253]. For example, NIRS-based brain-computer interface (BCI) systems, which establish a direct connection between the brain and an external device, have been successfully used in clinical trials of movement disorders and paralysis [254]. Neurofeedback applications, which provide clinical populations with (near) real-time information about their own brain functioning, have demonstrated long-term improvement in focused brain activity [255]. For patients with acquired or congenital brain insult, where elicitation of focused brain activity may be a crucial step toward complete neurorehabilitation, NIRSbased neurofeedback affords a potentially valuable treatment tool as well as an outcome measure. Thus, although not directly in FXS, data on NIRS supports its potential as a biomarker for evaluating shorter-term effects of interventions.

\section{Conclusions}

In conclusion, MRI techniques are potential biomarkers adequate for evaluating shorter- and longer-term effects. Due to their limited application in clinical trials in neurodevelopmental disorders, it is not possible at this point to assess the tool quality of MRI and other neuroimaging techniques. Nevertheless, due to its high cost and complexity, neuroimaging would be probably best employed in early-phase and target engagement studies in FXS.

\section{Overall conclusions on Biomarkers and Medical measures}

These are promising tools; however, the body of evidence supporting their use as outcome measures is still 
small. Despite this, the unique properties of biomarkers (e.g., objectivity, direct demonstration of target engagement) suggest that their inclusion in future studies developing endpoints or in early-phase intervention studies is critical.

\section{Other outcome measures}

Additional categories of outcome measures are being considered in FXS. The most advanced at present are instruments for assessing motor function, specifically the neuromotor battery under development by Tartaglia and colleagues [256].

In contrast to other neurodevelopmental disorders (e.g., Rett syndrome), impairments in FXS have been typically measured in a domain/area-specific function (e.g., language, anxiety). To date, no scales of overall clinical severity have been applied systematically to FXS. Nonetheless, a re-evaluation of the role of disorderspecific features and behavioral phenotype [3] is taking place, particularly in the context of clinical trials. For instance, the co-occurrence of different behavioral conditions (e.g., ASD and anxiety) in individuals with FXS modifies their presentation, evaluation, and interpretation [28]. Thus, the FXSRS [114], which covers a wide range of specific and common behavioral symptoms in FXS (discussed in the "Problem behaviors: focus on disruptive behavior domain" section), is the first attempt at developing a clinical severity scale for measuring response to interventions in FXS. Whether non-behavioral features should be added to measures such as the FXSRS will depend on the evaluation of their functional impact [43], a process at an early stage in FXS [17]. Indeed, as reviewed in the "Discussion" section, development and evaluation of quality of life measures in observational and intervention studies has just begun in FXS [257, 258].

\section{Discussion and conclusions}

FXS is a complex multi-system neurodevelopmental disorder. Its neurobehavioral manifestations, which include variable cognitive and language impairments, are linked to functional impairments and reduced quality of life [2]. FXS became a prototype neurodevelopmental disorder for developing neurobiologically targeted treatments (i.e., treatments targeting neurobiological abnormalities resulting from the primary genetic defect). Its wellcharacterized genetics, advanced neurobiological knowledge, and availability of animal models, in conjunction with increasing work on psychopharmacology, made FXS a focus of attention for federal funding and regulatory agencies and other stakeholders interested in developing new treatments. Two meetings led to the formation of Working Groups of experts and a published report on outcome measures in FXS [6]. Despite the relatively short interval between the 2013 Report (an updated version of the conclusions of the 2009 meeting) and the present review, the intense activity particularly in the field of targeted clinical trials and the apparent failure of all drug development programs that have advanced to phase IIb/III have made necessary this update on outcome measures in FXS.

The 2013 Report's overall conclusions were that in all three domains/areas, Cognition, Behavior/Emotion, and Biomarkers/Medical, there was a need for additional validation of existing and development of new measures for FXS. Of the available outcome measures, those covering the behavioral domain and in particular rating scales and questionnaires were closer to meeting all criteria for optimal endpoint. Specific recommendations were made for each area and type of measure. The present revision takes advantage of the additional experience collected in the last few years, a period in which further application of "older" measures and development and validation of newer instruments took place mainly in the context of ongoing intervention studies. Our overall conclusion is that the general and most of the specific recommendations of the 2013 Report are still valid [6]. Nonetheless, two areas have seen some steady progress: Cognition and Behavior/Emotion. In these fields, measures have been either adapted or developed specifically for FXS. With more ongoing and planned work on cognitive and behavioral instruments, the future seems encouraging. On the other hand, the Biomarkers/Medical area with its promise of providing more objective and quantitative tools has developed slowly, to some extent because of the reluctance of industry to embark on complicated and expensive projects that may not meet regulatory approval. The FXS clinical community can help with this process, by implementing in the clinic novel outcome measures, particularly those in the category of biomarkers. It is the continuous use and validation in the clinical setting that will make new tools more acceptable as meaningful endpoints. Thanks to the FXCRC, the FXS community is in a unique position for carrying out this endeavor.

Although the general conclusions of the present report suggest that optimal outcome measures for FXS are not yet fully developed, these conclusions should be seen as motivation for additional work [259, 260]. Recent similar efforts by the UK's NHS National Institute for Health Research [11] and Autism Speaks [13-15], which have evaluated mainly behavioral outcome measures for idiopathic ASD, have arrived at the same conclusions: few instruments meet validation criteria. The main question is whether this situation should lead to halting clinical trials while the shortcoming is remediated. While this may be an option for cognitive and behavioral trials, development of biomarkers is by nature slow, expensive, and more appropriate as a component of a clinical trial. 
Moreover, evaluating sensitivity of outcome measures is virtually impossible without their incorporation into intervention studies. Some information can be inferred from natural history studies and other investigations measuring change over time. However, the definitive answer can only be obtained from pragmatic (clinic practice-based) or conventional research clinical trials. The complications of sensitivity assessments are underscored by their dependence on treatment effectiveness (e.g., borderline effect of a drug at a given dose in FXS, but clear effect in another neurodevelopmental disorder). All of this means that refinement of existing and development of new outcome measures and biomarkers will be a continuing process incorporated to ongoing and future FXS trials and associated with surveillance of related work in other neurodevelopmental disorders [229]. This seems the only feasible approach; however, clinicians, scientists, participants, their families, and advocacy groups involved in FXS clinical trials should understand that, with this strategy, it is likely to take a number of trials to get study design worked out before a successful registration effort is achieved. Working closely with an informed FXS community seems the best strategy, if we continue moving forward with targeted trials while developing concurrently outcome measures.

An additional issue raised by the 2013 Report and the British and American efforts in ASD is the need to follow the recommendations of these reviews. Paraphrasing the 2013 Report, we should aim at "using a more consistent battery of measures across trials" in FXS. Clinical networks provide the ideal framework for the implementation of such batteries of outcome measures. The creation of a Clinical Trials Committee in the FXCRC could be seen as a step toward this goal. The committee is the most adequate entity for integrating clinical networks, such as the NIH-funded NeuroNEXT, drug companies, and other stakeholders involved in the development of clinical trials. FDA validation of an outcome measure for a given clinical trial has the benefit that such endpoint could be used in future studies, even if they do not involve the same drug. Thus, coordinated work among FXS clinical trial investigators may also lead to the availability of validated outcome measures for multiple clinical trials and for comparing the potency of different pharmacological and non-pharmacological interventions.

Finally, it is important to remember that in recent years, the FDA and its European counterpart, the European Medicines Agency, have emphasized the need not only to go beyond the measurement properties of endpoints but to also consider their meaning in terms of quality of life. In FXS, the common use of adaptive behavior instruments (e.g., Vineland-II) has helped to provide information on the potential functional implications of a "positive" trial
[6]. However, the systematic use of adaptive behavior and other more direct measures of quality of life in the validation of outcome measures in FXS would be an essential step as it has already been for other neurodevelopmental disorders [17]. Initial work with the ABC-C in FXS demonstrates the value of such endeavor [257, 258]. Ultimately, more solid outcome measures will benefit not only the development of new pharmacological interventions but of all types of treatments in FXS.

\section{Abbreviations}

ABC-C: Aberrant Behavior Checklist-Community Edition; ABC-C_I: ABC-C Irritability subscale; ABC-C_SW: ABC-C Lethargy/Social Withdrawal subscale; ABC-C_T: ABC-C total score; $A B C-C_{F x}$ : Aberrant Behavior Checklist-Community Edition FXS-specific; $A B C-C_{F X} S A$ : ABC- $C_{F X}$ Social Avoidance subscale; ACTeRS: Attention-Deficit Hyperactivity Comprehensive Teacher's Rating Scales; ADAMS: Anxiety Depression and Mood Scale; ADAMS_T: ADAMS total score; ADHD: Attention-deficit hyperactivity disorder; ADHD-RS: AttentionDeficit Hyperactivity Disorder Rating Scale; ADHD-RS-IV Ed: ADHD Rating Scale-IV; ADI-R: Autism Diagnostic Interview-Revised; ADIS: Anxiety Diagnostic Interview Scale for DSM-IV; ADOS: Autism Diagnostic Observation Schedule; ADOS-CSS: ADOS-Calibrated Severity Score; ADOS-G: ADOS-Generic;

AFQ056: Mavoglurant mGluR5 receptor antagonist; ALR: Allan L. Reiss, M.D.; APP: Amyloid precursor protein; ASD: Autism spectrum disorder; ATN: Autism Treatment Network; BASC: Behavioral Assessment for Children Scale; $\mathrm{BCl}$ : Brain-computer interface; BDNF: Brain-derived neurotrophic factor; BOLD: Blood oxygenation level dependent; BPI-S: Behavior Problems Inventory Short Form; CAG: Craig A. Erickson, M.D.; CARS: Childhood Autism Rating Scale; CASI-4R: Child and Adolescent Symptom Inventory-4th Ed Revised; CBCL: Child Behavior Checklist; CBI-M: Caregiver Burden InventoryModified; CELF-5/P2: Clinical Evaluation of Language Fundamentals-5th Ed/ Preschool-2nd Ed; CGI-I: Clinical Global Impression-Improvement; CGlS: Clinical Global Impression-Severity; CNS: Central nervous system; CNT: Contingency Naming Task; Conners Abb: Conners Abbreviated Version; Conners GI-P: Conners General Index-Parent Version; COSMIN: COnsensusbased Standards for the selection of health Measurement INstruments; CSHQ: Children's Sleep Habits Questionnaire; CSQ: Caregiver Strain Questionnaire; DAMP: Dextroamphetamine; DBB: Dejan B. Budimirovic, M.D.; DBC: Developmental Behavior Checklist; DH: David Hessl, Ph.D.;

DQ: Development quotient; DSM-5: Diagnostic and Statistical Manual of Mental Disorders-5th Edition; DSM-IV: Diagnostic and Statistical Manual of Mental Disorders-IVth Edition; DWI: Diffusion-weighted imaging; EBK: Elizabeth Berry-Kravis, M.D. Ph.D.; EEG: Electroencephalogram; EF: Executive function; ELS: Expressive language sampling; ERK: Extracellular signal-related kinases; ERP: Evoked response potentials; ET: Eye tracking; EVT2: Expressive Vocabulary Test 2nd ed; FDA: Food and Drug Administration; FMP: Fast-mapping performance; FMR1: Fragile $X$ mental retardation 1 gene; fMRI: Functional MRI; FMRP: Fragile X mental retardation protein; FSIQ: Fullscale intelligence quotient; FXCRC: Fragile X Clinical and Research Consortium; FXS: Fragile X syndrome; FXSRS: Fragile X Syndrome Rating Scale; FXTAS: Fragile X-associated tremor/ataxia syndrome; GABA: $g$ aminobutyric acid; HR: Heart rate; HRV: Heart rate variability; HSQ-PDD: Home Situations Questionnaire-PDDs version; ID: Intellectual disability; IGF-

1: Insulin-like growth factor; ILS: Independent Living Scales; IQ: Intelligence quotient; KiTAP: Tests of Attentional Performance for Children; LA: Leonard Abbeduto, Ph.D.; MAPK/ERK: Mitogen-activated protein kinases/extracellular signal-regulated kinases; MASC: Multidimensional Anxiety Scale for Children; MDX: Metadoxine; mGluR5: Metabotropic glutamate receptor 5; mGluRs: Group I metabotropic glutamate receptors; MKK: Margaret K. King; MMN: Mismatch negativity; MMP9: Matrix metallopeptidase 9; MPH: Methylphenidate; MRI: Magnetic resonance imaging; MRS: Magnetic resonance spectroscopy; MSEL: Mullen Scales of Early Learning; mTOR: mechanistic target of rapamycin; NICHD: National Institute of Child Health and Human Development; $\mathrm{NIH}$ : National Institutes of Health; $\mathrm{NIH}-$ TCB: NIH Toolbox Cognitive Battery; NIRS: Near-infrared spectroscopy; PARS: Pediatric Anxiety Rating Scale; PDD: Pervasive Developmental Disorders; PI: Principal Investigator; PK: Pharmacokinetics; PLS-5: Preschool Language Scale-Version 5; pMRI: Perfusion MRI; PPI: Prepulse inhibition; PPVT- 
4: Peabody Picture Vocabulary Test-4th Ed; PSI: Parenting Stress Index RBANS: Repeatable Battery for the Assessment of Neurological Status; RBQ: Repetitive Behavior Questionnaire; RBS-R: Repetitive Behavior ScaleRevised; RCADS: Revised Children's Anxiety and Depression Scale; RSA: Respiratory sinus arrhythmia; rs-fMRI: Resting state fMRI; sAPPa: Soluble neurotrophic amyloid precursor protein alpha; SB5: Stanford-Binet Version 5; SBS: Stereotyped Behavior Scale; SCARED: Screen for Child Anxiety Related Emotional Disorders; SCAS: Spence Children's Anxiety Scale; SCQ: Social Communication Questionnaire; SIB: Self-injurious behavior; sMRI: Structural MRI; SPPA: Self-Perception Profile for Adolescents; SRS: Social Responsiveness Scale; SRS-2: SRS 2nd Ed; SSH: Scott S. Hall, Ph.D.; SSRI: Selective serotonin reuptake inhibitor; Trofinetide: Neu-2566-FXS-001 study; VAS: Visual analog scale; VAS-C: VAS-clinician rated; VAS-mb: VAS-multiple behaviors; VASP: VAS-parent rated; VCFS: Velo-cardial-facial syndrome; Vineland-II: Vineland Adaptive Behavior Scales 2nd Ed; vMRI: Volumetric MRI; W-ADL: Waismen Activities of Daily Living Scale; WEK: Walter E. Kaufmann, M.D.; WIAT-II: Wechsler Individual Achievement Test-II; WISC-IV: Wechsler Intelligence Scale for Children 4th Ed; WISC-R: Wechsler Intelligence Scale for Children-Revised; WISC-V: Wechsler Intelligence Scale for Children 5th Ed; WJ: Woodcock Johnston; WM: Working memory tests; WPPSI-IV: Wechsler Preschool and Primary Scale of Intelligence 4th $\mathrm{Ed}$

\section{Acknowledgments}

The authors would like to acknowledge Duy Phan and Jayne Dixon Weber for their assistance with the tables and references and Dragana Protic, MD, PhD, for her assistance with the final endnote references and tables, as well as all of the coauthors. The authors would also like to thank the patients and families with fragile X syndrome for their participation and dedication to these studies; they are the inspiration for our efforts at improving treatments for the disorder.

\section{Funding}

While the authors are funded by Federal Agencies and private foundations, these sources were not applied to the writing of this manuscript.

\section{Availability of data and materials}

Not applicable.

\section{Authors' contributions}

DBB extensively reviewed the data and executed the design of this manuscript, participated in the interpretation of the data, then developed and steadily applied a grading system to measure the outcome measure tools, wrote the subsections and initially drafted the manuscript, developed and modified the tables throughout, critically revised the manuscript, generated references in endnote and applied them in the body text, and primarily coordinated all necessary efforts among coauthors on the manuscript throughout the process. EBK initiated and directed the design of this manuscript, participated in interpretation of the data and helped with development of the grading system, wrote the subsections of the manuscript and helped finalize them, coordinated efforts among other coauthors when necessary, and critically reviewed and revised the manuscript. CAE assisted with the design of the manuscript, wrote the subsections, critically reviewed and revised the manuscript, and chiefly coordinated its submission process. SSH assisted with the design of the manuscript, wrote and revised the subsections of the manuscript, and coordinated effort with other coauthors. DH assisted with the design of the manuscript, participated in the interpretation of the data, critically edited the "Cognitive measures" section of the manuscript, and coordinated effort with other coauthors. ALR assisted with design of the article, wrote and revised one of key subsections of the manuscript, and critically reviewed and revised the manuscript. MKK participated in gathering the reviewed literature, critically reviewed and revised the "Cognitive measures" section, and meaningfully coordinated an effort with other coauthors on the section. LA critically edited and wrote the subsections of the "Cognitive measures" section. WEK reviewed the data; assisted with design of the article; coordinated effort among authors; critically reviewed and revised the whole manuscript, determining its final content; and wrote the overall conclusions. All authors read and approved the final manuscript.

\section{Competing interests}

DBB has received support for clinical trials in FXS from Seaside Therapeutics and $\mathrm{NIH}$-research funding through Asuragen Inc. He has done an ad hock consulting work (rarely) for the American Academy of Child \& Adolescent Psychiatry, Ironshore, MEDACorp, Guidepoint and Sunovion.

EBK has received funding from Seaside Therapeutics, Novartis, Roche, Alcobra, Neuren, Cydan, and Neurotrope Pharmaceuticals to consult on trial design and development strategies and/or conduct clinical trials in FXS; from Vtesse to conduct clinical trials in NP-C; and from Asuragen Inc. to develop testing standards for FMR1 testing.

CAE has received research grant support from the National Institutes of Health, the United States Department of Defense, Autism Speaks, the Simons Foundation, and from the Cincinnati Children's Hospital Research Foundation. He is a current consultant to Fulcrum Therapeutics, Neurotrope, and Confluence Pharmaceuticals. He holds equity interest in Confluence Pharmaceuticals

SSH and ALR has no conflict of interests or disclosures.

$\mathrm{DH}$ has received funding from Seaside Therapeutics, Novartis, and Roche to consult on trial design and outcome measures in FXS clinical trials. MKK has no conflict of interests or disclosures at Novartis Pharmaceuticals. LA has received funding from the National Institutes of Health and financial support to develop and implement outcome measures for clinical trials from F. Hoffman-LaRoche, Ltd., Roche TCRC, Inc. and Neuren Pharmaceuticals. WEK has been a consultant for Neuren, Newron, Cydan, AstraZeneca, Edison, EryDel, Marinus, and GW Pharmaceuticals. He has received research support from Novartis and Ipsen.

\section{Consent for publication}

Not applicable.

\section{Ethics approval and consent to participate}

Not applicable.

\section{Author details}

'Departments of Psychiatry and Behavioral Sciences, Kennedy Krieger Institute and Child Psychiatry, Johns Hopkins University School of Medicine, 716 N. Broadway, Baltimore, MD 21205, USA. ${ }^{2}$ Departments of Pediatrics, Neurological Sciences, Biochemistry, Rush University Medical Center, 1725 West Harrison, Suite 718, Chicago, IL 60612, USA. ${ }^{3}$ Division of Child and Adolescent Psychiatry, Cincinnati Children's Hospital Medical Center and the University of Cincinnati College of Medicine, 3333 Burnet Avenue MLC 4002, Cincinnati, OH 45229, USA. ${ }^{4}$ Division of Interdisciplinary Brain Sciences, Department of Psychiatry and Behavioral Sciences, Stanford University, 401 Quarry Road, Stanford, CA 94305, USA. ${ }^{5}$ MIND Institute and Department of Psychiatry and Behavioral Sciences, University of California Davis Medical Center, 2825 50th Street, Sacramento, CA 95817, USA. ${ }^{6}$ Division of Interdisciplinary Brain Sciences, Departments of Psychiatry and Behavioral Sciences, Radiology and Pediatrics, Stanford University, 401 Quarry Road, Stanford, CA 94305, USA. ${ }^{7}$ Autism \& Developmental Medicine Institute, Geisinger Health System, Present address: Novartis Pharmaceuticals Corporation, US Medical, One Health Plaza, East Hanover, NJ 07936, USA. ${ }^{8}$ MIND Institute and Department of Psychiatry and Behavioral Sciences, University of California Davis Medical Center, 2825 50th Street, Sacramento, CA 95817, USA. ${ }^{9}$ Center for Translational Research, Greenwood Genetic Center, 113 Gregor Mendel Circle, Greenwood, SC 29646, USA. ${ }^{10}$ Department of Neurology, Boston Children's Hospital, Boston, MA 02115, USA.

Received: 27 July 2016 Accepted: 22 February 2017

Published online: 12 June 2017

\section{References}

1. Kaufmann WE, Reiss AL. Molecular and cellular genetics of fragile $X$ syndrome. Am J Med Genet. 1999;88:11-24.

2. Hagerman RJ, Berry-Kravis E, Kaufmann WE, Ono MY, Tartaglia N, Lachiewicz A, Kronk R, Delahunty C, Hessl D, Visootsak J, et al. Advances in the treatment of fragile $X$ syndrome. Pediatrics. 2009;123:378-90.

3. Boyle L, Kaufmann WE. The behavioral phenotype of FMR1 mutations. Am J Med Genet. 2010;154C:469-76.

4. Schaefer TL, Davenport MH, Erickson CA. Emerging pharmacologic treatment options for fragile X syndrome. Appl Clin Genet. 2015;8:75-93.

5. Hampson DR, Gholizadeh S, Pacey LKK. Pathways to drug development for autism spectrum disorders. Clin Pharmacol Ther. 2011;91:189-200. 
6. Berry-Kravis E, Hessl D, Abbeduto L, Reiss AL, Beckel-Mitchener A, Urv TK. Outcome measures for clinical trials in fragile $X$ syndrome. J Dev Behav Pediatr. 2013;34:508-22.

7. Davenport MH, Schaefer TL, Friedmann KJ, Fitzpatrick SE, Erickson CA Pharmacotherapy for fragile X syndrome: progress to date. Drugs. 2016;76: 431-45.

8. Mullard A. Fragile X disappointments upset autism ambitions. Nat Rev Drug Discov. 2015;14:151-3.

9. Berry-Kravis E, Des Portes V, Hagerman R, Jacquemont $S$, Charles P, Visootsak J, Brinkman M, Rerat K, Koumaras B, Zhu L, et al. Mavoglurant in fragile X syndrome: results of two randomized, double-blind, placebo-controlled trials. Sci Transl Med. 2016:8:321ra325.

10. Wigham S, McConachie H. Systematic review of the properties of tools used to measure outcomes in anxiety intervention studies for children with autism spectrum disorders. PLoS One. 2014;9:e85268.

11. McConachie H, Parr JR, Glod M, Hanratty J, Livingstone N, Oono IP, Robalino S, Baird G, Beresford B, Charman T, et al. Systematic review of tools to measure outcomes for young children with autism spectrum disorder Health Technol Assess. 2015;19:1-506.

12. Hanratty J, Livingstone N, Robalino S, Terwee CB, Glod M, Oono IP, Rodgers J, Macdonald G, McConachie H. Systematic review of the measurement properties of tools used to measure behaviour problems in young children with autism. PLoS One. 2015;10:e0144649.

13. Lecavalier L, Wood JJ, Halladay AK, Jones NE, Aman MG, Cook EH, Handen BL, King $\mathrm{BH}$, Pearson DA, Hallett V, et al. Measuring anxiety as a treatment endpoint in youth with autism spectrum disorder. J Autism Dev Disord. 2013:44:1128-43.

14. Anagnostou E, Jones N, Huerta M, Halladay AK, Wang P, Scahill L, Horrigan JP, Kasari C, Lord C, Choi D, et al. Measuring social communication behaviors as a treatment endpoint in individuals with autism spectrum disorder. Autism. 2014;19:622-36.

15. Scahill L, Aman MG, Lecavalier L, Halladay AK, Bishop SL, Bodfish JW, Grondhuis S, Jones N, Horrigan JP, Cook EH, et al. Measuring repetitive behaviors as a treatment endpoint in youth with autism spectrum disorder. Autism. 2015;19:38-52

16. Budimirovic DB, Kaufmann WE. What can we learn about autism from studying fragile X syndrome? Dev Neurosci. 2011;33:379-94.

17. Barnes KV, Coughlin FR, O'Leary HM, Bruck N, Bazin GA, Beinecke EB, Walco AC, Cantwell NG, Kaufmann WE. Anxiety-like behavior in Rett syndrome: characteristics and assessment by anxiety scales. J Neurodev Disord. 2015;7:30.

18. Kaufmann WE, Cortell R, Kau AS, Bukelis I, Tierney E, Gray RM, Cox C, Capone GT, Stanard P. Autism spectrum disorder in fragile $X$ syndrome: communication, social interaction, and specific behaviors. Am J Med Genet A. 2004;129A:225-34

19. Budimirovic DB, Bukelis I, Cox C, Gray RM, Tierney E, Kaufmann WE. Autism spectrum disorder in fragile $X$ syndrome: differential contribution of adaptive socialization and social withdrawal. Am J Med Genet A. 2006;140A:1814-26.

20. Abbeduto L, Kover S, McDuffie A. Handbook of child language research methods. 2012.

21. Berry-Kravis E, Doll E, Sterling A, Kover ST, Schroeder SM, Mathur S, Abbeduto L. Development of an expressive language sampling procedure in fragile X syndrome: a pilot study. J Dev Behav Pediatr 2013;34:245-51

22. Roid GH. Stanford-Binet intelligence scales. Itasca: Riverside Publishing; 2003 .

23. Wechsler D, Coalson DL, Raiford SE. Wechsler adult intelligence scale-fourthWAIS-IV. San Antonio: Pearson; 2008.

24. Wahlstrom D, Breaux KC, Zhu J, Weiss LG. The Wechsler preschool and primary scale of intelligence-third edition, the Wechsler Intelligence Scale for Children_fourth edition, and the Wechsler individual achievement test. In: Flanagan DP, Harrison PL, editors. Contemporary intellectual assessment: theories, tests, and issues. New York: Guilford Press; 2012. p. 224-8.

25. Wechsler D. Wechsler Intelligence Scale for Children-fifth edition (WISC-V). San Antonio: The Psychological Corporation; 2014.

26. Mullen EM. Mullen scales of early learning. Circle Pines: AGS; 1995

27. Bayley N, Reuner G. Bayley scales of infant and toddler development: Bayley-III. San Antonio: Harcourt Assessment, Psych. Corporation; 2006.

28. Kaufmann WE, Capone GT, Clarke M, Budimirovic DB. Autism in genetic intellectual disability. In: Autism. Totowa: The Humana Press Inc; 2008. p. 81-108

29. Klaiman C, Quintin E-M, Jo B, Lightbody AA, Hazlett HC, Piven J, Hall SS, Reiss AL. Longitudinal profiles of adaptive behavior in fragile $X$ syndrome. Pediatrics. 2014. doi: 10.1542/peds.2013-3990.
30. Sparrow SS, Cicchetti DV, Balla DA. Vineland Adaptive Behavior Scales: Vineland II), survey interview form/caregiver rating form. 2005.

31. Sparrow S, Cicchetti D, Saulnier C. Vineland Adaptive Behavior Scales, third edition (Vineland-3). 2016.

32. Hessl D, Nguyen DV, Green C, Chavez A, Tassone F, Hagerman RJ, Senturk D, Schneider A, Lightbody A, Reiss AL, Hall S. A solution to limitations of cognitive testing in children with intellectual disabilities: the case of fragile X syndrome. J Neurodev Disord. 2009;1:33-45.

33. Sansone SM, Schneider A, Bickel E, Berry-Kravis E, Prescott C, Hessl D. Improving IQ measurement in intellectual disabilities using true deviation from population norms. J Neurodev Disord. 2014;6:16.

34. Quintin EM, Jo B, Hall SS, Bruno JL, Chromik LC, Raman MM, Lightbody AA, Martin A, Reiss AL. The cognitive developmental profile associated with fragile $X$ syndrome: a longitudinal investigation of cognitive strengths and weaknesses through childhood and adolescence. Dev Psychopathol. 2016; 28:1457-69

35. Akshoomoff N, Newman E, Thompson WK, McCabe C, Bloss CS, Chang L, Amaral DG, Casey BJ, Ernst TM, Frazier JA, et al. The NIH toolbox cognition battery: results from a large normative developmental sample (PING). Neuropsychology. 2014;28:1-10.

36. Gershon RC, Wagster MV, Hendrie HC, Fox NA, Cook KF, Nowinski CJ. NIH toolbox for assessment of neurological and behavioral function. Neurology. 2013;80:S2-6.

37. Casaletto KB, Umlauf A, Beaumont J, Gershon R, Slotkin J, Akshoomoff N, Heaton RK. Demographically corrected normative standards for the English version of the $\mathrm{NIH}$ toolbox cognition battery. J Int Neuropsychol Soc. 2015; 21:378-91.

38. Hessl D, Sansone SM, Berry-Kravis E, Riley K, Widaman KF, Abbeduto L, Schneider A, Coleman J, Oaklander D, Rhodes KC. The NIH toolbox cognitive battery for intellectual disabilities: three preliminary studies and future directions. J Neurodev Disord. 2016;8:35.

39. Association AP. Diagnostic and statistical manual of mental disorders (DSM- $5^{\oplus}$ ). Washington: American Psychiatric Pub; 2013.

40. Berry-Kravis E, Krause SE, Block SS, Guter S, Wuu J, Leurgans S, Decle P, Potanos K, Cook E, Salt J, et al. Effect of CX516, an AMPA-modulating compound, on cognition and behavior in fragile $X$ syndrome: a controlled trial. J Child Adolesc Psychopharmacol. 2006;16:525-40.

41. Perry A, Flanagan HE, Geier JD, Freeman NL. Brief report: the Vineland Adaptive Behavior Scales in young children with autism spectrum disorders at different cognitive levels. J Autism Dev Disord. 2009;39:1066-78.

42. Duker P, Driel S, Bercken J. Communication profiles of individuals with Down's syndrome, Angelman syndrome and pervasive developmental disorder. J Intellect Disabil Res. 2002:46:35-40.

43. Fidler D, Hepburn S, Rogers S. Early learning and adaptive behaviour in toddlers with Down syndrome: evidence for an emerging behavioural phenotype? Down Syndrome Research and Practice. 2006:9:37-44

44. Berry-Kravis E. A 6-week, randomized, multicenter, double-blind, parallel, flexed-and fixed-dose study of MDX (metadoxine extended-release: $\mathrm{MG01Cl}$ ) compared with placebo in adolescents and adults with fragile $X$ syndrome. In: 62nd Annual Meeting. San Antonio: AACAP; 2015.

45. Wang P, Wang L, Rathmell B, Carpenter R, Hagerman R, Berry-Kravis E. Treatment of fragile X syndrome with STX209 (arbaclofen): open label extension experience. Toronto: IMFAR; 2012.

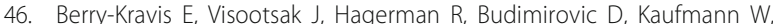
Bear M, Walton-Bowen K, Carpenter R, Wang P. Arbaclofen in fragile X syndrome: results of phase 3 trials. In: Ann Neurol. Hoboken: Wiley-Blackwell; 2014. p. S174.

47. McCary LM, Machlin L, Roberts JE. The development of adaptive behaviour in toddlers and preschoolers with fragile $X$ versus autism. Int J Dev Disab. 2013;59:67-79.

48. Frolli A, Piscopo S, Conson M. Developmental changes in cognitive and behavioural functioning of adolescents with fragile-X syndrome. J Intellect Disabil Res. 2015:59:613-21.

49. de Bildt A, Kraijer D, Sytema S, Minderaa R. The psychometric properties of the Vineland Adaptive Behavior Scales in children and adolescents with mental retardation. J Autism Dev Disord. 2005;35:53-62.

50. Woodcock RW, Mather N, McGrew KS. Woodcock-Johnson III tests of cognitive abilities examiner's manual. Itasca: Riverside Publishing; 2001.

51. Woodcock RR, McGrew KS, Schrank FA. Woodcock-Johnson III normative update technical manual. Itasca: Riverside Publishing; 2007. 
52. Kessels RPC, Van Zandvoort MJE, Postma A, Kappelle L, De Haan EHF. The Corsi block-tapping task: standardization and normative data. Appl Neuropsychol. 2000;7:252-8.

53. Randolph C. Repeatable battery for the assessment of neuropsychological status (RBANS). San Antonio: Psychological Corporation; 1998.

54. Berry-Kravis E, Sumis A, Kim OK, Lara R, Wuu J. Characterization of potentia outcome measures for future clinical trials in fragile $X$ syndrome. J Autism Dev Disord. 2008;38:1751-7.

55. Berry-Kravis E, Sumis A, Hervey C, Nelson M, Porges SW, Weng N, Weiler IJ, Greenough WT. Open-label treatment trial of lithium to target the underlying defect in fragile X syndrome. J Dev Behav Pediatr. 2008;29:293-302.

56. Galusha-Glasscock JM, Horton DK, Weiner MF, Cullum CM. Video teleconference administration of the repeatable battery for the assessment of neuropsychological status. Arch Clin Neuropsychol. 2016; 31:8-11.

57. Wilding J, Cornish K, Munir F. Further delineation of the executive deficit in males with fragile-X syndrome. Neuropsychologia. 2002;40:1343-9.

58. Scerif G, Cornish K, Wilding J, Driver J, Karmiloff-Smith A. Delineation of early attentional control difficulties in fragile $X$ syndrome: focus on neurocomputational changes. Neuropsychologia. 2007;45:1889-98.

59. Hooper SR, Hatton D, Sideris J, Sullivan K, Hammer J, Schaaf J, Mirrett P, Ornstein PA, Bailey Jr DP. Executive functions in young males with fragile $X$ syndrome in comparison to mental age-matched controls: baseline findings from a longitudinal study. Neuropsychology. 2008;22:36-47.

60. Zimmermann P, Fimm B, Gondan M. Testbatterie zur aufmerksamkeitsprüfung für kinder:(KiTAP);[das schloß der geister]. Frankfurt: Psytest; 2002.

61. Konrad K, Gunther T, Hanisch C, Herpertz-Dahlmann B. Differential effects of methylphenidate on attentional functions in children with attention-deficit/hyperactivity disorder. J Am Acad Child Adolesc Psychiatry. 2004;43:191-8.

62. Knox A, Schneider A, Abucayan F, Hervey C, Tran C, Hessl D, Berry-Kravis E. Feasibility, reliability, and clinical validity of the test of attentional performance for children (KiTAP) in fragile X syndrome (FXS). J Neurodev Disord. 2012:4:2

63. Neuren. Neuren's trofinetide successful in proof of concept phase 2 clinical trial in fragile X syndrome, vol. 2016. 2015.

64. Alcobra P. Alcobra announces results from phase 2 clinical trial of MDX for fragile $x$ syndrome. 2015

65. Gioia GA, Isquith PK, Guy SC, Kenworthy L. Behavior rating inventory of executive function. Child Neuropsychol. 2000;6:235-8.

66. Abbeduto L, Brady N, Kover ST. Language development and fragile $X$ syndrome: Profiles, syndrome-specificity, and within-syndrome differences. Ment Retard Dev Disabil Res Rev. 2007;13:36-46.

67. Dunn DM, Dunn LM. Peabody picture vocabulary test: manual. San Antonio: Pearson; 2007.

68. Williams KT. EVT-2: Expressive vocabulary test. San Antonio: Pearson Assessments; 2007

69. Wiig EH, Secord W, Semel EM. CELF preschool 2: clinical evaluation of language fundamentals preschool. Pearson/PsychCorp; 2004.

70. Wiig E, Semel E, Secord W. Clinical evaluation of language fundamentals ${ }^{\oplus}$ — fifth edition (CELF ${ }^{\oplus}$-5). San Antonio: Pearson/PsychCorp.; 2013.

71. Dollaghan C. Child meets word: "fast mapping" in preschool children. J Speech Hear Res. 1985;28:449-54.

72. McDuffie A, Kover ST, Hagerman R, Abbeduto L. Investigating word learning in fragile $X$ syndrome: a fast-mapping study. J Autism Dev Disord. 2013;43:1676-91.

73. Benjamin DP, McDuffie AS, Thurman AJ, Kover ST, Mastergeorge AM, Hagerman RJ, Abbeduto L. Effect of speaker gaze on word learning in fragile $X$ syndrome: a comparison with nonsyndromic autism spectrum disorder. J Speech Lang Hear Res. 2015;58:383-95.

74. Thurman AJ, McDuffie A, Kover ST, Hagerman R, Channell MM, Mastergeorge A, Abbeduto L. Use of emotional cues for lexical learning: a comparison of autism spectrum disorder and fragile $X$ syndrome. J Autism Dev Disord. 2015:45:1042-61.

75. Benjamin D, Mastergeorge A, McDuffie A, Kover S, Hagerman R, Abbeduto $L$. Effects of labeling and pointing on object gaze in boys with fragile $X$ syndrome: an eye-tracking study. Res Dev Disabil. 2014;35:2658-72.

76. Kover ST, McDuffie A, Abbeduto L, Brown WT. Effects of sampling context on spontaneous expressive language in males with fragile $X$ syndrome or Down syndrome. J Speech Lang Hear Res. 2012;55:1022-38.
77. Hall SS, Frank MC, Pusiol GT, Farzin F, Lightbody AA, Reiss AL. Quantifying naturalistic social gaze in fragile $X$ syndrome using a novel eye tracking paradigm. Am J Med Genet B Neuropsychiatr Genet. 2015; 168:564-72.

78. Constantino JN, Gruber CP. The Social Responsiveness Scale (SRS) manual. Los Angeles: Western Psychological Services; 2007.

79. Constantino JN, Todd RD. Genetic structure of reciprocal social behavior. Am J Psychiatry. 2000;157:2043-5.

80. Constantino JN, Gruber CP, Davis S, Hayes S, Passanante N, Przybeck T. The factor structure of autistic traits. J Child Psychol Psychiatry. 2004;45:719-26.

81. Bolte S, Poustka F, Constantino JN. Assessing autistic traits: cross-cultural validation of the Social Responsiveness Scale (SRS). Autism Res. 2008;1:354-63.

82. Duku E, Vaillancourt T, Szatmari P, Georgiades S, Zwaigenbaum L, Smith IM, Bryson S, Fombonne E, Mirenda P, Roberts W, et al. Investigating the measurement properties of the Social Responsiveness Scale in preschool children with autism spectrum disorders. J Autism Dev Disord. 2013;43:860-8.

83. Pine E, Luby J, Abbacchi A, Constantino JN. Quantitative assessment of autistic symptomatology in preschoolers. Autism. 2006;10:344-52.

84. Constantino JN, Abbacchi AM, Lavesser PD, Reed H, Givens L, Chiang L, Gray T, Gross M, Zhang Y, Todd RD. Developmental course of autistic social impairment in males. Dev Psychopathol. 2009;21:127-38.

85. Farzin F, Scaggs F, Hervey C, Berry-Kravis E, Hessl D. Reliability of eye tracking and pupillometry measures in individuals with fragile $X$ syndrome. J Autism Dev Disord. 2011;41:1515-22.

86. Crawford H, Moss J, Anderson GM, Oliver C, McCleery JP. Implicit discrimination of basic facial expressions of positive/negative emotion in fragile $X$ syndrome and autism spectrum disorder. Am J Intellect Dev Disabil. 2015;120:328-45.

87. Leigh MJ, Nguyen DV, Mu Y, Winarni TI, Schneider A, Chechi T, Polussa J, Doucet $\mathrm{P}$, Tassone F, Rivera SM, et al. A randomized double-blind, placebocontrolled trial of minocycline in children and adolescents with fragile $x$ syndrome. J Dev Behav Pediatr. 2013;34:147-55.

88. Sansone S, Berry-Kravis E, Crestodina C, McLennan Y, Hagerman R, Hessl D. The sensitivity of an eye tracking paradigm as an outcome measure in a fragile $x$ syndrome targeted treatment clinical trial. In: San Diego, CA. 49th Gatlinburg Conference on Research and Theory in Intellectual Disabilities. 2016.

89. Turkstra LS, Abbeduto L, Meulenbroek P. Social cognition in adolescent girls with fragile x syndrome. Am J Intellect Dev Disabil. 2014;119:319-39.

90. Baron-Cohen S, Wheelwright S, Scahill V, Lawson J, Spong A. Are intuitive physics and intuitive psychology independent? A test with children with Asperger syndrome. J Dev Learn Disord. 2001;5:47-78.

91. Klusek J, Hunt AW, Mirrett PL, Hatton DD, Hooper SR, Roberts JE, Bailey DB. Reading and phonological skills in boys with fragile $X$ syndrome. J Autism Dev Disord. 2015;45:1699-711.

92. Hall SS, Hammond JL, Hirt M, Reiss AL. A "learning platform" approach to outcome measurement in fragile $X$ syndrome: a preliminary psychometric study. J Intellect Disabil Res. 2012;56:947-60.

93. Hall SS, Hustyi KM, Hammond JL, Hirt M, Reiss AL. Using discrete trial training to identify specific learning impairments in boys with fragile $X$ syndrome. J Autism Dev Disord. 2014:44:1659-70.

94. Au J, Berkowitz-Sutherland L, Schneider A, Schweitzer JB, Hessl D, Hagerman R. A feasibility trial of Cogmed working memory training in fragile X syndrome. J Pediatr Genet. 2014;3:147-56.

95. McDuffie A, Machalicek W, Bullard L, Nelson S, Mello M, TemperoFeigles R, Castignetti N, Abbeduto L. A spoken-language intervention for school-aged boys with fragile $X$ syndrome. Am J Intellect Dev Disabil. 2016;121:236-65.

96. McDuffie A, Oakes A, Machalicek W, Ma M, Bullard L, Nelson S, Abbeduto L. Early language intervention using distance video-teleconferencing: a pilot study of young boys with fragile $X$ syndrome and their mothers. Am J Speech Lang Pathol. 2016;25:46-66.

97. Loeb PA. ILS: Independent Living Scales manual. San Antonio: Psychological Corporation; 1996.

98. Hustyi KM, Hall SS, Quintin E-M, Chromik LC, Lightbody AA, Reiss AL. The relationship between autistic symptomatology and independent living skills in adolescents and young adults with fragile X syndrome. J Autism Dev Disord. 2015:45:1836-44.

99. Martin A, Quintin EM, Hall S, Reiss AL. The role of executive function in independent living skills in female adolescents and young adults with fragile X syndrome. Am J Intellect Dev Disabil. 2016;121:448-60. 
100. Maenner MJ, Smith LE, Hong J, Makuch R, Greenberg JS, Mailick MR. Evaluation of an activities of daily living scale for adolescents and adults with developmental disabilities. Disabil Health J. 2013;6:8-17.

101. Smith LE, Hong J, Greenberg JS, Mailick MR. Change in the behavioral phenotype of adolescents and adults with FXs: role of the family environment. J Autism Dev Disord. 2016:46:1824-33.

102. Bruininks RH, Woodcock RW, Weatherman RE, Hill BK. Scales of Independent Behavior-Revised (SIB-R) Riverside. 1996

103. Wheeler A, Raspa M, Bann C, Bishop E, Hessl D, Sacco P, Bailey Jr DB. Anxiety, attention problems, hyperactivity, and the Aberrant Behavior Checklist in fragile X syndrome. Am J Med Genet A. 2014;164A:141-55.

104. Aman MG, Singh NN, Stewart AW, Field CJ. Psychometric characteristics of the Aberrant Behavior Checklist. Am J Ment Defic. 1985;89:492-502.

105. McCracken JT, McGough J, Shah B, Cronin P, Hong D, Aman MG, Arnold LE, Lindsay R, Nash P, Hollway J, et al. Risperidone in children with autism and serious behavioral problems. N Engl J Med. 2002;347:314-21.

106. Zarcone JR, Hellings JA, Crandall K, Reese RM, Marquis J, Fleming K, Shores R, Williams D, Schroeder SR. Effects of risperidone on aberrant behavior of persons with developmental disabilities: I. A double-blind crossover study using multiple measures. Am J Ment Retard. 2001;106:525-38.

107. Arnold LE, Vitiello B, McDougle C, Scahill L, Shah B, Gonzalez NM, Chuang S, Davies M, Hollway J, Aman MG, et al. Parent-defined target symptoms respond to risperidone in RUPP autism study: customer approach to clinical trials. J Am Acad Child Adolesc Psychiatry. 2003;42:1443-50.

108. McDougle CJ, Scahill L, McCracken JT, Aman MG, Tierney E, Arnold LE, Freeman BJ, Martin A, McGough JJ, Cronin P, et al. Research units on pediatric psychopharmacology (RUPP) autism network. Background and rationale for an initial controlled study of risperidone. Child Adolesc Psychiatr Clin N Am. 2000;9:201-24.

109. Erickson CA, Stigler KA, Wink LK, Mullett JE, Kohn A, Posey DJ, McDougle CJ. A prospective open-label study of aripiprazole in fragile $X$ syndrome. Psychopharmacology (Berl). 2011;216:85-90

110. Fung LK, Quintin EM, Haas BW, Reiss AL. Conceptualizing neurodevelopmental disorders through a mechanistic understanding of fragile $X$ syndrome and Williams syndrome. Curr Opin Neurol. 2012;25:112-24.

111. Sansone SM, Widaman KF, Hall SS, Reiss AL, Lightbody A, Kaufmann WE Berry-Kravis E, Lachiewicz A, Brown EC, Hessl D. Psychometric study of the Aberrant Behavior Checklist in fragile $X$ syndrome and implications for targeted treatment. J Autism Dev Disord. 2012;42:1377-92.

112. Berry-Kravis EM, Hessl D, Rathmell B, Zarevics $P$, Cherubini M, Walton-Bowen $K$, Mu Y, Nguyen DV, Gonzalez-Heydrich J, Wang PP, et al. Effects of STX209 (arbaclofen) on neurobehavioral function in children and adults with fragile $X$ syndrome: a randomized, controlled, phase 2 trial. Sci Transl Med. 2012;4:152ra127.

113. Berry-Kravis E, Hagerman RJ, Visootsak J, Budimirovic DB, Kaufmann WE, Bear MF, Walton-Bowen K, Wang P, Carpenter RL. Arbaclofen in fragile X syndrome: results of phase 3 trials. J Neurodev Disord. 2016. (in press JNDD-D-16-00048R2).

114. Snape M, Horrigan J, Glass L, et al. Improving outcome measures for fragile $X$ syndrome clinical trials: development of fragile $X$ syndrome specific rating scales. In: Society for the Study of Behavioral Phenotypes New York City. 2014.

115. Rojahn J, Rowe EW, Sharber AC, Hastings R, Matson JL, Didden R, Kroes DB, Dumont EL. The behavior problems inventory-short form for individuals with intellectual disabilities: part I: development and provisional clinical reference data. J Intellect Disabil Res. 2012;56:527-45.

116. Rojahn J, Matson JL, Lott D, Esbensen AJ, Smalls Y. The behavior problems inventory: an instrument for the assessment of self-injury, stereotyped behavior, and aggression/destruction in individuals with developmental disabilities. J Autism Dev Disord. 2001;31:577-88.

117. Hessl D, Berry-Kravis E, Cordeiro L, Yuhas J, Ornitz EM, Campbell A, Chruscinski E, Hervey C, Long JM, Hagerman RJ. Prepulse inhibition in fragile $X$ syndrome: feasibility, reliability, and implications for treatment. Am J Med Genet B Neuropsychiatr Genet. 2009;150B:545-53.

118. Bailey Jr DB, Raspa M, Olmsted M, Holiday DB. Co-occurring conditions associated with FMR1 gene variations: findings from a national parent survey. Am J Med Genet A. 2008;146A:2060-9.

119. Reilly C, Senior J, Murtagh L. ASD, ADHD, mental health conditions and psychopharmacology in neurogenetic syndromes: parent survey. J Intellect Disabil Res. 2015:59:307-18.

120. DuPaul GJ, Anastopoulos AD, Power TJ, Reid R, Ikeda MJ, McGoey KE. Parent ratings of attention-deficit/hyperactivity disorder symptoms: factor structure and normative data. J Psychopathol Behav Assess. 1998;20:83-102.
121. Association AP. Diagnostic and statistical manual of mental disorders (DSM) —IVth edition. Washington DC: APA; 1994.

122. Zhang $S$, Faries DE, Vowles $M$, Michelson D. ADHD rating scale IV: psychometric properties from a multinational study as a clinicianadministered instrument. Int J Methods Psychiatr Res. 2005;14:186-201.

123. Gilliam JE. Attention-deficit/hyperactivity disorder test: a method for identifying individuals with ADHD. Austin: Pro-ed; 1995.

124. Conners CK, Wells KC, Parker JD, Sitarenios G, Diamond JM, Powell JW. A new self-report scale for assessment of adolescent psychopathology: factor structure, reliability, validity, and diagnostic sensitivity. J Abnorm Child Psychol. 1997;25:487-97.

125. Chromik LC, Quintin EM, Lepage JF, Hustyi KM, Lightbody AA, Reiss AL. The influence of hyperactivity, impulsivity, and attention problems on social functioning in adolescents and young adults with fragile $X$ syndrome. J Atten Disord. 2015. [Epub ahead of print].

126. Cornish K, Cole V, Longhi E, Karmiloff-Smith A, Scerif G. Mapping developmental trajectories of attention and working memory in fragile $X$ syndrome: developmental freeze or developmental change? Dev Psychopathol. 2013;25:365-76.

127. Achenbach T, Rescorla L. Manual for the ASEBA school-age forms \& profiles: an integrated system of multi-informant assessment Burlington, $\mathrm{VT}$ : University of Vermont. Burlington: Research Center for Children, Youth, \& Families; 2001.

128. Achenbach TM. Manual for the child behavior checklist/4-18 and 1991 profile. Burlington: Department of Psychiatry, University of Vermont; 1991.

129. Nakamura BJ, Ebesutani C, Bernstein A, Chorpita BF. A psychometric analysis of the child behavior checklist DSM-oriented scales. J Psychopathol Behav Assess. 2009;31:178-89.

130. Grefer M, Flory K, Cornish K, Hatton D, Roberts J. The emergence and stability of attention deficit hyperactivity disorder in boys with fragile $X$ syndrome. J Intellect Disabil Res. 2016:60:167-78.

131. Swanson JM, Sandman CA, Deutsch C, Baren M. Methylphenidate hydrochloride given with or before breakfast: I. Behavioral, cognitive, and electrophysiologic effects. Pediatrics. 1983;72:49-55.

132. Cordeiro L, Ballinger E, Hagerman R, Hessl D. Clinical assessment of DSM-IV anxiety disorders in fragile $X$ syndrome: prevalence and characterization. J Neurodev Disord. 2011;3:57-67.

133. Esbensen AJ, Rojahn J, Aman MG, Ruedrich S. Reliability and validity of an assessment instrument for anxiety, depression, and mood among individuals with mental retardation. J Autism Dev Disord. 2003;33:617-29.

134. Group RAS. The Pediatric Anxiety Rating Scale (PARS): development and psychometric properties. J Am Acad Child Adolesc Psychiatry. 2002:41:1061-9.

135. Talisa VB, Boyle L, Crafa D, Kaufmann WE. Autism and anxiety in males with fragile $X$ syndrome: an exploratory analysis of neurobehavioral profiles from a parent survey. Am J Med Genet A. 2014;164A:1198-203.

136. Tonnsen BL, Malone PS, Hatton DD, Roberts JE. Early negative affect predicts anxiety, not autism, in preschool boys with fragile $X$ syndrome. J Abnorm Child Psychol. 2013;41:267-80.

137. Tonnsen BL, Cornish KM, Wheeler AC, Roberts JE. Maternal predictors of anxiety risk in young males with fragile X. Am J Med Genet B Neuropsychiatr Genet. 2014;165B:399-409.

138. Russo-Ponsaran NM, Yesensky J, Hessl D, Berry-Kravis E. Feasibility, reproducibility, and clinical validity of the pediatric anxiety rating scalerevised for fragile $X$ syndrome. Am J Intellect Dev Disabil. 2014;119:1-16.

139. Khwaja OS, Ho E, Barnes KV, O'Leary HM, Pereira LM, Finkelstein Y, Nelson 3rd CA, Vogel-Farley V, DeGregorio G, Holm IA, et al. Safety, pharmacokinetics, and preliminary assessment of efficacy of mecasermin (recombinant human IGF-1) for the treatment of Rett syndrome. Proc Nat Acad Sci U S A. 2014:111:4596-601.

140. Budimirovic DB, Haas B, Blitz R, Esler A, Kaufmann WE, Sudhalter V, Stackhouse T, Berry-Kravis $E$. Consensus of the fragile $X$ clinical and research consortium on clinical practices autism spectrum disorder in fragile X syndrome. Walnut Creek, California: National Fragile X Foundation Website; 2014

141. Kaufmann WE, Kidd SA, Andrews H, Budimirovic DB, Esler A, Haas B, Stackhouse T, Peacock G, Riley C, Sherman SL, et al. Autism spectrum disorder in fragile $X$ syndrome: characterization using the fragile $X$ online registry with accessible research database (FORWARD). Pediatrics. 2015. (in press). 
142. Mayes SD, Calhoun SL. Analysis of WISC-III, Stanford-Binet: IV, and academic achievement test scores in children with autism. J Autism Dev Disord. 2003; 33:329-41.

143. Mayes SD, Calhoun SL. Ability profiles in children with autism: influence of age and IQ. Autism. 2003;7:65-80.

144. Hernandez RN, Feinberg RL, Vaurio R, Passanante NM, Thompson RE, Kaufmann WE. Autism spectrum disorder in fragile X syndrome: a longitudinal evaluation. Am J Med Genet A. 2009;149A:1125-37.

145. Hartley SL, Wheeler AC, Mailick MR, Raspa M, Mihaila I, Bishop E, Bailey DB. Autism symptoms across adulthood in men with fragile $X$ syndrome: a cross-sectional analysis. J Autism Dev Disord. 2015;45:3668-79.

146. Hatton DD, Sideris J, Skinner M, Mankowski J, Bailey Jr DB, Roberts J, Mirrett $P$. Autistic behavior in children with fragile $X$ syndrome: prevalence, stability, and the impact of FMRP. Am J Med Genet A. 2006;140A:1804-13.

147. Lord C, Risi S, Lambrecht L, Cook Jr EH, Leventhal BL, DiLavore PC, Pickles A, Rutter M. The autism diagnostic observation schedule-generic: a standard measure of social and communication deficits associated with the spectrum of autism. J Autism Dev Disord. 2000;30:205-23.

148. Gotham K, Pickles A, Lord C. Standardizing ADOS scores for a measure of severity in autism spectrum disorders. J Autism Dev Disord. 2009;39:693-705.

149. Dawson G, Rogers S, Munson J, Smith M, Winter J, Greenson J, Donaldson A, Varley J. Randomized, controlled trial of an intervention for toddlers with autism: the Early Start Denver Model. Pediatrics. 2010;125:e17-23.

150. Green J, Charman T, McConachie H, Aldred C, Slonims V, Howlin P, Le Couteur A, Leadbitter K, Hudry K, Byford S, et al. Parent-mediated communication-focused treatment in children with autism (PACT): a randomised controlled trial. Lancet. 2010;375:2152-60.

151. de Bildt A, Oosterling IJ, van Lang ND, Sytema S, Minderaa RB, van Engeland H, Roos S, Buitelaar JK, van der Gaag R-J, de Jonge MV. Standardized ADOS scores: measuring severity of autism spectrum disorders in a Dutch sample. J Autism Dev Disord. 2011:41:311-9.

152. Shumway S, Farmer C, Thurm A, Joseph L, Black D, Golden C. The ADOS calibrated severity score: relationship to phenotypic variables and stability over time. Autism Res. 2012;5:267-76.

153. Lord C, Risi S, DiLavore PS, Shulman C, Thurm A, Pickles A. Autism from 2 to 9 years of age. Arch Gen Psychiatry. 2006;63:694-701.

154. Kamp-Becker I, Ghahreman M, Smidt J, Remschmidt H. Dimensional structure of the autism phenotype: relations between early development and current presentation. J Autism Dev Disord. 2009;39:557-71.

155. Luyster R, Gotham K, Guthrie W, Coffing M, Petrak R, Pierce K, Bishop S, Esler A, Hus V, Oti R, et al. The autism diagnostic observation schedule-toddler module: a new module of a standardized diagnostic measure for autism spectrum disorders. J Autism Dev Disord. 2009;39:1305-20.

156. Chawarska K, Klin A, Paul R, Volkmar F. Autism spectrum disorder in the second year: stability and change in syndrome expression. J Child Psychol Psychiatry. 2007;48:128-38.

157. Gray K, Tonge B, Sweeney D, Einfeld S. Screening for autism in young children with developmental delay: an evaluation of the developmental behaviour checklist: early screen. J Autism Dev Disord. 2008:38:1003-10

158. Norris M, Lecavalier L, Edwards MC. The structure of autism symptoms as measured by the autism diagnostic observation schedule. J Autism Dev Disord. 2012;42:1075-86.

159. Erickson CA, Wink LK, Ray B, Early MC, Stiegelmeyer E, Mathieu-Frasier L, Patrick V, Lahiri DK, McDougle CJ. Impact of acamprosate on behavior and brain-derived neurotrophic factor: an open-label study in youth with fragile X syndrome. Psychopharmacology (Berl). 2013;228:75-84.

160. Schopler E, Reichler RJ, DeVellis RF, Daly K. Toward objective classification of childhood autism: Childhood Autism Rating Scale (CARS). J Autism Dev Disord. 1980;10:91-103.

161. Schopler E, Reichler RJ, Renner BR. Child autism rating scale. Los Angeles: Western Psychological Services Corporation; 1988.

162. Schopler E, Van Bourgondien ME, Wellman GJ, Love SR. CARS 2: Childhood Autism Rating Scale. 2010.

163. Charman T, Howlin P, Berry B, Prince E. Measuring developmental progress of children with autism spectrum disorder on school entry using parent report. Autism. 2004;8:89-100

164. Russell PS, Daniel A, Russell S, Mammen P, Abel JS, Raj LE, Shankar SR, Thomas N. Diagnostic accuracy, reliability and validity of Childhood Autism Rating Scale in India. World J Pediatr. 2010;6:141-7.
165. Stella J, Mundy P, Tuchman R. Social and nonsocial factors in the Childhood Autism Rating Scale. J Autism Dev Disord. 1999;29:307-17.

166. Magyar Cl, Pandolfi V. Factor structure evaluation of the Childhood Autism Rating Scale. J Autism Dev Disord. 2007;37:1787-94.

167. Berument SK, Rutter M, Lord C, Pickles A, Bailey A. Autism screening questionnaire: diagnostic validity. Br J Psychiatry. 1999;175:444-51.

168. Lord C, Rutter M, Le Couteur A. Autism diagnostic interview-revised: a revised version of a diagnostic interview for caregivers of individuals with possible pervasive developmental disorders. J Autism Dev Disord. 1994;24:659-85.

169. Cochran L, Moss J, Nelson L, Oliver C. Contrasting age related changes in autism spectrum disorder phenomenology in Cornelia de Lange, fragile $\mathrm{X}$, and Cri du Chat syndromes: results from a 2.5 year follow-up. In: American Journal of Medical Genetics Part C: Seminars in Medical Genetics. Hoboken: John Wiley \& Sons, Inc; 2015. p. 188-197.

170. Hall SS, Lightbody AA, Hirt M, Rezvani A, Reiss AL. Autism in fragile X syndrome: a category mistake? J Am Acad Child Adolesc Psychiatry. 2010;49:921-33.

171. Moss J, Oliver C, Nelson L, Richards C, Hall S. Delineating the profile of autism spectrum disorder characteristics in Cornelia de Lange and fragile $X$ syndromes. Am J Intellect Dev Disabil. 2013;118:55-73.

172. Waite J, Moss J, Beck SR, Richards C, Nelson L, Arron K, Burbidge C, Berg K, Oliver C. Repetitive behavior in Rubinstein-Taybi syndrome: parallels with autism spectrum phenomenology. J Autism Dev Disord. 2015;45:1238-53.

173. Snow AV, Lecavalier L. Sensitivity and specificity of the modified checklist for autism in toddlers and the social communication questionnaire in preschoolers suspected of having pervasive developmental disorders. Autism. 2008;12:627-44.

174. Magyar Cl, Pandolfi V, Dill CA. An initial evaluation of the social communication questionnaire for the assessment of autism spectrum disorders in children with Down syndrome. J Dev Behav Pediatr. 2012;33:134-45.

175. Turner M. Annotation: repetitive behaviour in autism: a review of psychological research. J Child Psychol Psychiatry. 1999;40:839-49.

176. Bishop SL, Richler J, Lord C. Association between restricted and repetitive behaviors and nonverbal IQ in children with autism spectrum disorders. Child Neuropsychol. 2006;12:247-67.

177. Richler J, Huerta M, Bishop SL, Lord C. Developmental trajectories of restricted and repetitive behaviors and interests in children with autism spectrum disorders. Dev Psychopathol. 2010;22:55-69.

178. Green VA, Sigafoos J, Pituch KA, Itchon J, O'Reilly M, Lancioni GE. Assessing behavioral flexibility in individuals with developmental disabilities. Focus Autism Other Dev Disabl. 2006:21:230-6.

179. Bodfish JW, Symons FJ, Parker DE, Lewis MH. Varieties of repetitive behavior in autism: comparisons to mental retardation. J Autism Dev Disord. 2000;30:237-43.

180. Lam KS, Bodfish JW, Piven J. Evidence for three subtypes of repetitive behavior in autism that differ in familiality and association with other symptoms. J Child Psychol Psychiatry. 2008:49:1193-200.

181. Lam KS, Aman MG. The repetitive behavior scale-revised: independent validation in individuals with autism spectrum disorders. J Autism Dev Disord. 2007;37:855-66.

182. Mirenda P, Smith IM, Vaillancourt T, Georgiades S, Duku E, Szatmari P, Bryson S, Fombonne E, Roberts W, Volden J. Validating the repetitive behavior scale-revised in young children with autism spectrum disorder J Autism Dev Disord. 2010;40:1521-30.

183. Bishop SL, Hus V, Duncan A, Huerta M, Gotham K, Pickles A, Kreiger A, Buja A Lund S, Lord C. Subcategories of restricted and repetitive behaviors in children with autism spectrum disorders. J Autism Dev Disord. 2013;43:1287-97.

184. Harrop C, McConachie H, Emsley R, Leadbitter K, Green J, Consortium P. Restricted and repetitive behaviors in autism spectrum disorders and typical development: cross-sectional and longitudinal comparisons. J Autism Dev Disord. 2014:44:1207-19.

185. Brock M, Hatton D. Distinguishing features of autism in boys with fragile $X$ syndrome. J Intellect Disabil Res. 2010;54:894-905.

186. Wolff JJ, Bodfish JW, Hazlett HC, Lightbody AA, Reiss AL, Piven J. Evidence of a distinct behavioral phenotype in young boys with fragile $X$ syndrome and autism. J Am Acad Child Adolesc Psychiatry. 2012;51:1324-32.

187. Wolff JJ, Hazlett HC, Lightbody AA, Reiss AL, Piven J. Repetitive and selfinjurious behaviors: associations with caudate volume in autism and fragile X syndrome. J Neurodev Disord. 2013;5:1.

188. Lewis M, Kim SJ. The pathophysiology of restricted repetitive behavior. J Neurodev Disord. 2009;1:114-32. 
189. Moss J, Oliver C, Arron K, Burbidge C, Berg K. The prevalence and phenomenology of repetitive behavior in genetic syndromes. J Autism Dev Disord. 2009;39:572-88.

190. Edwards MJ, Lang AE, Bhatia KP. Stereotypies: a critical appraisal and suggestion of a clinically useful definition. Mov Disord. 2012;27:179-85.

191. Sudhalter V, Cohen IL, Silverman W, Wolf-Schein EG. Conversational analyses of males with fragile $X$, Down syndrome, and autism: comparison of the emergence of deviant language. Am J Ment Retard. 1990;94(4):431-41.

192. Roberts JE, Chapman RS, Warren SF, Brookes PH. Language of preschool and school-age children with Down syndrome and fragile $X$ syndrome. In: Roberts JE, Chapman RS, Warren SF, editors. Speech and language development and intervention in Down syndrome and Fragile $X$ syndrome. Baltimore: Brookes Pub; 2008

193. Hagerman RJ. The physical and behavioral phenotype. In: Hagerman RJ, Hagerman PJ, editors. Fragile X syndrome: diagnosis, treatment, and research. Baltimore: Johns Hopkins University Press; 2002.

194. Oakes A, Thurman A, McDuffie A, Bullard L, Hagerman R, Abbeduto L. Characterising repetitive behaviours in young boys with fragile $X$ syndrome. J Intellect Disabil Res. 2016;60:54-67.

195. Rojahn J. Self-injurious and stereotypic behavior of noninstitutionalized mentally retarded people: prevalence and classification. Am J Ment Defic. 1986:30:49-57.

196. Bartak L, Rutter M. Differences between mentally retarded and normally intelligent autistic children. J Autism Child Schizophr. 1976;6:109-20.

197. Valenza G, Nardelli M, Lanata A, Gentili C, Bertschy G, Paradiso R, Scilingo EP. Wearable monitoring for mood recognition in bipolar disorder based on history-dependent long-term heart rate variability analysis. IEEE J Biomed Health Inform. 2014;18:1625-35.

198. Bakker D, Kazantzis N, Rickwood D, Rickard N. Mental health smartphone apps: review and evidence-based recommendations for future developments. JMIR Mental Health. 2016;3(1):e7.

199. Weng N, Weiler IJ, Sumis A, Berry-Kravis E, Greenough WT. Early-phase ERK activation as a biomarker for metabolic status in fragile $\mathrm{X}$ syndrome. Am J Med Genet B Neuropsychiatr Genet. 2008;147:1253-7.

200. Erickson CA, Weng N, Weiler IJ, Greenough WT, Stigler KA, Wink LK, McDougle CJ. Open-label riluzole in fragile X syndrome. Brain Res. 2011; 1380:264-70.

201. Berridge MJ. Inositol trisphosphate and calcium signalling. Nature. 1993;361: 315-25.

202. Doble A. The pharmacology and mechanism of action of riluzole. Neurology. 1996;47:233S-41.

203. Pellerin D, Çaku A, Fradet M, Bouvier P, Dubé J, Corbin F. Lovastatin corrects ERK pathway hyperactivation in fragile $X$ syndrome: potential of platelet's signaling cascades as new outcome measures in clinical trials. Biomarkers. 2016;21(6):497-508.

204. Castrén M, Lampinen KE, Miettinen R, Koponen E, Sipola I, Bakker CE, Oostra BA, Castrén E. BDNF regulates the expression of fragile $X$ mental retardation protein mRNA in the hippocampus. Neurobiol Dis. 2002;11: 221-9.

205. Lauterborn JC, Rex CS, Kramár E, Chen LY, Pandyarajan V, Lynch G, Gall CM. Brain-derived neurotrophic factor rescues synaptic plasticity in a mouse model of fragile X syndrome. In: The Journal of Neuroscience, vol. 27. 2007. p. 10685-94.

206. Uutela M, Lindholm J, Louhivuori V, Wei H, Louhivuori L, Pertovaara A Åkerman K, Castrén E, Castrén M. Reduction of BDNF expression in Fmr1 knockout mice worsens cognitive deficits but improves hyperactivity and sensorimotor deficits. Genes Brain Behav. 2012;11:513-23.

207. Kiefer F, Mann K. Acamprosate: how, where, and for whom does it work? Mechanism of action, treatment targets, and individualized therapy. Curr Pharm Des. 2010;16:2098-102.

208. Makkonen I, Riikonen R, Kuikka JT, Kokki H, Bressler JP, Marshall C, Kaufmann WE. Brain derived neurotrophic factor and serotonin transporter binding as markers of clinical response to fluoxetine therapy in children with autism. J Pediatr Neurol. 2011;9:1-8.

209. Lahiri DK, Sokol DK, Erickson C, Ray B, Ho CY, Maloney B. Autism as early neurodevelopmental disorder: evidence for an SAPPa-mediated anabolic pathway. Front Cell Neurosci. 2013;7:94.

210. De Rubeis S, Bagni C. Fragile X mental retardation protein control of neuronal mRNA metabolism: insights into mRNA stability. Mol Cell Neurosci. 2010;43:43-50
211. Westmark CJ, Malter JS. FMRP mediates mGluR 5-dependent translation of amyloid precursor protein. PLoS Biol. 2007:5:e52

212. Theoharides TC, Stewart JM, Panagiotidou S, Melamed I. Mast cells, brain inflammation and autism. Eur J Pharmacol. 2016;778:96-102.

213. Theoharides T, Tsilioni I, Patel A, Doyle R. Atopic diseases and inflammation of the brain in the pathogenesis of autism spectrum disorders. Transl Psychiatry. 2016;6:e844.

214. Gu F, Chauhan V, Chauhan A. Glutathione redox imbalance in brain disorders. Curr Opin Clin Nutr Metab Care. 2015;18:89-95.

215. Muller C, Anacker A, Veenstra-VanderWeele J. The serotonin system in autism spectrum disorder: from biomarker to animal models. Neuroscience. 2016;321:24-41.

216. Farzin F, Rivera SM, Hessl D. Brief report: visual processing of faces in individuals with fragile $X$ syndrome: an eye tracking study. J Autism Dev Disord. 2009:39:946-52.

217. Niedermeyer E, da Silva FHL. Electroencephalography: basic principles, clinical applications, and related fields. Philadelphia: Lippincott Williams \& Wilkins; 2005

218. Pizzagalli DA. Electroencephalography and high-density electrophysiological source localization. Handbook of psychophysiology. 2007;3:56-84.

219. Van der Molen M, Van der Molen M, Ridderinkhof K, Hamel B, Curfs L, Ramakers $G$. Auditory change detection in fragile $X$ syndrome males: a brain potential study. Clin Neurophysiol. 2012;123:1309-18.

220. Miller L, McIntosh D, McGrath J, Shyu V, Lampe M, Taylor A, Tassone F, Neitzel K, Stackhouse T, Hagerman RJ. Electrodermal responses to sensory stimuli in individuals with fragile X syndrome. Am J Med Genet. 1999;83: 268-79.

221. Galvez R, Gopal AR, Greenough WT. Somatosensory cortical barrel dendritic abnormalities in a mouse model of the fragile $X$ mental retardation syndrome. Brain Res. 2003;971:83-9.

222. Rotschafer S, Razak K. Altered auditory processing in a mouse model of fragile X syndrome. Brain Res. 2013;1506:12-24.

223. Gandal MJ, Edgar JC, Ehrlichman RS, Mehta M, Roberts TP, Siegel SJ. Validating $\mathrm{Y}$ oscillations and delayed auditory responses as translational biomarkers of autism. Biol Psychiatry. 2010;68:1100-6.

224. Harvey B, Siok C, Kiss T, Volfson D, Grimwood S, Shaffer C, Hajós M. Neurophysiological signals as potential translatable biomarkers for modulation of metabotropic glutamate 5 receptors. Neuropharmacology. 2013;75:19-30.

225. Roberts TP, Cannon KM, Tavabi K, Blaskey L, Khan SY, Monroe JF, Qasmieh S, Levy SE, Edgar JC. Auditory magnetic mismatch field latency: a biomarker for language impairment in autism. Biol Psychiatry. 2011;70:263-9.

226. Berry-Kravis E, Hessl D, Coffey S, Hervey C, Schneider A, Yuhas J, Hutchison J, Snape M, Tranfaglia M, Nguyen DV. A pilot open label, single dose trial of fenobam in adults with fragile $X$ syndrome. J Med Genet. 2009;46:266-71.

227. Schneider A, Leigh MJ, Adams P, Nanakul R, Chechi T, Olichney J, Hagerman $R$, Hess I D. Electrocortical changes associated with minocycline treatment in fragile $X$ syndrome. J Psychopharmacol. 2013;27:956-63.

228. Yang J-C, Niu Y-Q, Simon C, Seritan AL, Chen L, Schneider A, Moghaddam ST, Hagerman PJ, Hagerman RJ, Olichney JM. Memantine effects on verbal memory in fragile X-associated tremor/ataxia syndrome (FXTAS): a double-blind brain potential study. Neuropsychopharmacology. 2014:39:2760-8.

229. Gross C, Hoffmann A, Bassell GJ, Berry-Kravis EM. Therapeutic strategies in fragile $X$ syndrome: from bench to bedside and back. Neurotherapeutics. 2015;12:584-608.

230. Ethridge L, White S, Mosconi M, Wang J, Byerly M, Sweeney J. Reduced habituation of auditory evoked potentials indicate cortical hyper-excitability in fragile X syndrome. Transl Psychiatry. 2016;6:e787.

231. Dunn W. Sensory profile 2 manual. San Antonio: Pearson; 2014

232. Van Hecke AV, Stevens S, Carson AM, Karst JS, Dolan B, Schohl K, McKindles RJ, Remmel R, Brockman S. Measuring the plasticity of social approach: a randomized controlled trial of the effects of the PEERS intervention on EEG asymmetry in adolescents with autism spectrum disorders. J Autism Dev Disord. 2015:45:316-35.

233. Wang Y, Sokhadze EM, El-Baz AS, Li X, Sears L, Casanova MF, Tasman A. Relative power of specific EEG bands and their ratios during neurofeedback training in children with autism spectrum disorder. Front Hum Neurosci. $2015 ; 9$. 
234. Mueller S, Schuff N, Weiner M. Evaluation of treatment effects in Alzheimer's and other neurodegenerative diseases by MRI and MRS. NMR Biomed. 2006; 19:655-68.

235. Fung LK, Reiss AL. Moving toward integrative, multidimensional research in modern psychiatry: lessons learned from fragile $X$ syndrome. Biol Psychiatry. 2016;80(2):100-11.

236. Brown TT, Kuperman JM, Chung Y, Erhart M, McCabe C, Hagler DJ, Venkatraman VK, Akshoomoff N, Amaral DG, Bloss CS. Neuroanatomical assessment of biological maturity. Curr Biol. 2012;22:1693-8.

237. Roberto CA, Mayer LE, Brickman AM, Barnes A, Muraskin J, Yeung LK, Steffener J, Sy M, Hirsch J, Stern Y. Brain tissue volume changes following weight gain in adults with anorexia nervosa. Int J Eat Disord. 2011:44:406-11.

238. Eijk J, Demirakca T, Frischknecht U, Hermann D, Mann K, Ende G. Rapid partial regeneration of brain volume during the first 14 days of abstinence from alcohol. Alcohol Clin Exp Res. 2013;37:67-74.

239. Garver DL, Holcomb JA, Christensen JD. Cerebral cortical gray expansion associated with two second-generation antipsychotics. Biol Psychiatry. 2005; 58:62-6.

240. Arnone D, Mckie S, Elliott R, Juhasz G, Thomas E, Downey D, Williams S, Deakin J, Anderson I. State-dependent changes in hippocampal grey matter in depression. Mol Psychiatry. 2013;18:1265-72.

241. Ibrahim I, Tintera J, Skoch A, Jirů F, Hlustik P, Martinkova P, Zvara K, Rasova K. Fractional anisotropy and mean diffusivity in the corpus callosum of patients with multiple sclerosis: the effect of physiotherapy. Neuroradiology. 2011;53:917-26

242. Wang T, Huang X, Huang P, Li D, Lv F, Zhang Y, Zhou L, Yang D, Xie P. Early-stage psychotherapy produces elevated frontal white matter integrity in adult major depressive disorder. PLoS One. 2013;8:e63081.

243. Fan Q, Yan X, Wang J, Chen Y, Wang X, Li C, Tan L, You C, Zhang T, Zuo S. Abnormalities of white matter microstructure in unmedicated obsessivecompulsive disorder and changes after medication. PLoS One. 2012;7: e35889

244. Matthews PM, Honey GD, Bullmore ET. Applications of fMRI in translational medicine and clinical practice. Nat Rev Neurosci. 2006;7:732-44.

245. Chapin H, Bagarinao E, Mackey S. Real-time fMRI applied to pain management. Neurosci Lett. 2012;520:174-81.

246. Singh MK, Chang KD. The neural effects of psychotropic medications in children and adolescents. Child Adolesc Psychiatr Clin N Am. 2012;21: 753-71.

247. Beauregard M. Functional neuroimaging studies of the effects of psychotherapy. Dialogues Clin Neurosci. 2014;16:75.

248. Lukas SE. New perspectives on using brain imaging to study CNS stimulants. Neuropharmacology. 2014;87:104-14.

249. Gudayol-Ferré E, Peró-Cebollero M, González-Garrido AA, Guàrdia-Olmos J. Changes in brain connectivity related to the treatment of depression measured through fMRI: a systematic review. Front Hum Neurosci. 2015;9:582.

250. Reid LB, Boyd RN, Cunnington R, Rose SE. Interpreting intervention induced neuroplasticity with $\mathrm{fMRI}$ : the case for multimodal imaging strategies. Neural Plast. 2015:2016:1-13.

251. Strangman G, Culver JP, Thompson JH, Boas DA. A quantitative comparison of simultaneous BOLD fMRI and NIRS recordings during functional brain activation. Neuroimage. 2002;17:719-31.

252. Obrig H. NIRS in clinical neurology—a "promising"tool? Neuroimage. 2014; 85:535-46.

253. Cutini S, Brigadoi S. Unleashing the future potential of functional nearinfrared spectroscopy in brain sciences. J Neurosci Methods. 2014;232:152-6.

254. Birbaumer N, Murguialday AR, Cohen L. Brain-computer interface in paralysis. Curr Opin Neurol. 2008;21:634-8.

255. Kober SE, Wood G, Kurzmann J, Friedrich EV, Stangl M, Wippel T, Väljamäe A, Neuper C. Near-infrared spectroscopy based neurofeedback training increases specific motor imagery related cortical activation compared to sham feedback. Biol Psychol. 2014;95:21-30.

256. Tartaglia N, Cordeiro L, McGrew J, Stackhouse T. Neuromotor outcome measures for clinical trials in fragile $\mathrm{x}$ syndrome. In: 13th International Fragile X Conference. Miami, FL. 2012

257. Raspa M, Sacco P, Candrilli S, Bishop E, Petrillo J. Validity of a condition specific outcome measure for fragile $X$ syndrome: the Aberrant Behaviour Checklist-utility index. J Intellect Disabil Res. 2016:60(9):844-55.
258. Kerr C, Breheny K, Lloyd A, Brazier J, Bailey Jr DB, Berry-Kravis E, Cohen J, Petrillo J. Developing a utility index for the Aberrant Behavior Checklist (ABC-C) for fragile X syndrome. Qual Life Res. 2015;24:305-14.

259. Budimirovic DB, Duy PQ. Challenges in translating therapeutic frontiers in clinical trials: where are we now and what's next. Madridge J Neuro Sci. 2016;1:e1-3.

260. Jeste SS, Geschwind DH. Clinical trials for neurodevelopmental disorders: at a therapeutic frontier. Sci Transl Med. 2016;8:321fs321.

261. Jacquemont S, Curie A, des Portes V, Torrioli MG, Berry-Kravis E, Hagerman RJ, Ramos FJ, Cornish K, He Y, Paulding C, et al. Epigenetic modification of the FMR1 gene in fragile $X$ syndrome is associated with differential response to the mGluR5 antagonist AFQ056. Sci Transl Med. 2011;3:64ra61.

262. Greiss-Hess L, Hagerman RJ. Effects of sertraline treatment for young children with fragile $X$ syndrome: examination of developmental outcomes and family contexts. Dissertation Abstracts International Section A: Humanities and Social Sciences. U California, Davis. 2015.

263. Hall SS, Lightbody AA, McCarthy BE, Parker KJ, Reiss AL. Effects of intranasal oxytocin on social anxiety in males with fragile $X$ syndrome. Psychoneuroendocrinology. 2012;37:509-18.

264. de Diego-Otero Y, Calvo-Medina R, Quintero-Navarro C, Sánchez-Salido L, García-Guirado F, del Arco-Herrera I, Fernández-Carvajal I, Ferrando-Lucas T, Caballero-Andaluz R, Pérez-Costillas L. A combination of ascorbic acid and a-tocopherol to test the effectiveness and safety in the fragile $X$ syndrome: study protocol for a phase II, randomized, placebo-controlled trial. Trials. 2014;15:1.

265. Wirojanan J, Jacquemont S, Diaz R, Bacalman S, Anders TF, Hagerman RJ, Goodlin-Jones BL. The efficacy of melatonin for sleep problems in children with autism, fragile $X$ syndrome, or autism and fragile $X$ syndrome. J Clin Sleep Med. 2009:5:145-50.

266. Torrioli MG, Vernacotola S, Peruzzi L, Tabolacci E, Mila M, Militerni $R$, Musumeci S, Ramos FJ, Frontera M, Sorge G, et al. A double-blind, parallel, multicenter comparison ofL-acetylcarnitine with placebo on the attention deficit hyperactivity disorder in fragile X syndrome boys. Am J Med Genet 2008;146A:803-12.

267. Hagerman RJ, Murphy MA, Wittenberger MD. A controlled trial of stimulant medication in children with the fragile X syndrome. Am J Med Genet. 1988; 30:377-92.

268. Budimirovic D, Phan D. Neurobehavioral features and targeted treatments in fragile $X$ syndrome: current insights and future directions. Engrams. 2015; 37:5-19.

269. Hoeffer CA, Sanchez E, Hagerman RJ, Mu Y, Nguyen DV, Wong H, Whelan AM, Zukin RS, Klann E, Tassone F. Altered mTOR signaling and enhanced CYFIP2 expression levels in subjects with fragile $X$ syndrome. Genes Brain Behav. 2012;11:332-41.

270. Kesler SR, Lightbody AA, Reiss AL. Cholinergic dysfunction in fragile $X$ syndrome and potential intervention: a preliminary $1 \mathrm{H}$ MRS study. Am J Med Genet A. 2009;149A:403-7.

\section{Submit your next manuscript to BioMed Central and we will help you at every step:}

- We accept pre-submission inquiries

- Our selector tool helps you to find the most relevant journal

- We provide round the clock customer support

- Convenient online submission

- Thorough peer review

- Inclusion in PubMed and all major indexing services

- Maximum visibility for your research

Submit your manuscript at www.biomedcentral.com/submit 